\title{
Application of Deep Learning in Dentistry and Implantology
}

\author{
Dae-Young Kang, DDS, MSD', Hieu Pham Duong, DDS, MSD, PhD², Jung-Chul Park, DDS, \\ MSD, $\mathrm{PhD}^{3 *}$ \\ ${ }^{1}$ Clinical Assistant Professor, Department of Periodontology, Dankook University College of Dentistry, Cheonan, \\ Korea \\ ${ }^{2}$ Associate Dean, Department of Maxillo-Stomatology, Vietnam National University School of Medicine and \\ Pharmacy, Hanoi, Vietnam \\ ${ }^{3}$ Associate Professor, Department of Periodontology, Dankook University College of Dentistry, Cheonan, Korea \\ *Corresponding author: Jung-Chul Park, Professor, Department of Periodontology, Dankook University \\ College of Dentistry, 119 Dandae-ro, Dongnam-gu, Cheonan 31116, Korea. \\ Tel: +82-41-550-1931. Fax: +82-303-3442-7364. E-mail: jcp@dent.dku.edu
}

\section{OPEN ACCESS}

pISSN 1229-5418

eISSN 2671-6623

Implantology 2020; 24(3): 148-181

https://doi.org/10.32542/implantology.202015

Received: June 9, 2020

Revised: July 25,2020

Accepted: July 29, 2020

ORCID

Dae-Young Kang

https://orcid.org/0000-0002-4311-4118

Hieu Pham Duong

https://orcid.org/0000-0001-5409-0361

Jung-Chul Park

https://orcid.org/0000-0002-2041-8047

Copyright $($ 2020. The Korean Academy of Oral \& Maxillofacial Implantology

\footnotetext{
This is an Open Access article distributed under the terms of the Creative Commons Attribution Non-Commercial License (http://creativecommons. org/licenses/by-nc/4.0/) which permits unrestricted non-commercial use, distribution, and reproduction in any medium, provided the original work is properly cited.
}

\begin{abstract}
Artificial intelligence and deep learning algorithms are infiltrating various fields of medicine and dentistry. The purpose of the current study was to review literatures applying deep learning algorithms to the dentistry and implantology. Electronic literature search through MEDLINE and IEEE Xplore library database was performed at 2019 October by combining free-text terms and entry terms associated with 'dentistry' and 'deep learning'. The searched literature was screened by title/abstract level and full text level. Following data were extracted from the included studies: information of author, publication year, the aim of the study, architecture of deep learning, input data, output data, and performance of the deep learning algorithm in the study. 340 studies were retrieved from the databases and 62 studies were included in the study. Deep learning algorithms were applied to tooth localization and numbering, detection of dental caries/periodontal disease/ periapical disease/oral cancerous lesion, localization of cephalometric landmarks, image quality enhancement, prediction and compensation of deformation error in additive manufacturing of prosthesis. Convolutional neural network was used for periapical radiograph, panoramic radiograph, or computed tomography in most of included studies. Deep learning algorithms are expected to help clinicians diagnose and make decisions by extracting dental data, detecting diseases and abnormal lesions, and improving image quality.
\end{abstract}

Keywords: Deep learning; Dentistry; Dental implants; Machine learning; Neural networks; Radiography, Dental

\section{I . Introduction}

The Go competition between the artificial intelligence (AI) AlphaGo of Google DeepMind and the legendary Go player Lee Sedol-in which AlphaGo won 4:1straightforwardly shows the development of AI. At present, AI is being widely used in 
various fields including SPAM mail filters, search algorithms and ranking system of web search engines, facial recognition algorithms of social networking services, and personalized curation algorithms of contents or products (Fig. 1). ${ }^{1}$ Alibaba achieved daily sales $\$ 38$ billion during the Singles Day in 2019, $26 \%$ higher than the previous year, by launching an AI fashion assistant which has been trained about hundreds of millions of clothes. Amazon is automating most of their logistics except packing and is managing "Amazon Go" checkout-free convenience store chain in the US. In the Amazon Go, the payment is automatically processed when customers exit with their products based on real-time location tracking of them using multiple cameras, weight measuring sensors, and deep learning algorithms.

$\mathrm{AI}$ is expected to have huge impact on the healthcare industry. Currently, more than 40 and 10 deep learning algorithms have been approved as medical devices by the US Food and Drug Administration (FDA) and Ministry of Food and Drug Safety (MFDS) in South Korea, respectively. For example, fourth-generation Apple Watch and AliveCor KardiaMobile with deep learning algorithm have been approved by the US FDA as over-the-counter medical devices for detecting atrial fibrillation. These algorithms show an accuracy for detecting abnormal findings comparable to that of humans by training hundreds of thousands of data.

\section{Gartner Hype Cycle for Artificial Intelligence, 2019}

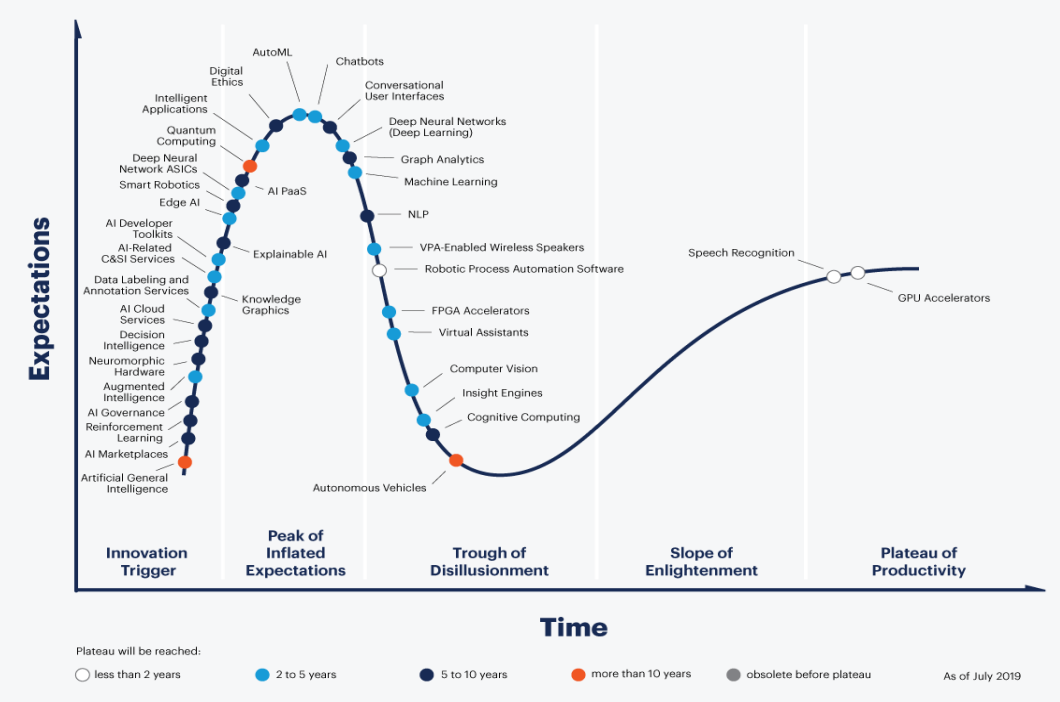

gartner.com/SmarterWithGartner

Source: Gartner

Gartner.

Fig. 1. Hype cycle for artificial intelligence 2019. Reprinted from "Gartner Hype Cycle for Artificial Intelligence 2019" by Kenneth Brant, Jim Hare, Svetlana Sicular, Copyright 2019 by Gartner, Inc. and/or its affiliates. https://www.gartner.com/smarterwithgartner/top-trends-on-the-gartner-hype-cycle-forartificial-intelligence-2019/ 
Dentistry is a field of study that requires a high level of accuracy; it is expected that AI and deep learning algorithms will be introduced in the near future and provide great assistance to clinical practices. In South Korea, an algorithm that estimates bone age from a hand-wrist radiograph has been approved by the MFDS; however, not many other cases have been reported yet. Therefore, this study aims to examine the global trends of deep learning technologies applied to dentistry and to forecast the future of dentistry.

\section{II . Materials and Methods}

\section{Literature Search}

To select literature on the application of deep learning algorithms in dentistry, we searched the MEDLINE and IEEE Xplore databases for papers in all languages that were published before October 24,2019 . The search formula was set up by combining free-text term and entry term about the deep learning, neural network, and dentistry (Table 1).

\section{Selection of Papers}

Papers were selected in two steps: first, papers were selected based on their title and abstract; second, their full text was evaluated. The criteria for selecting papers were as follows: (1) papers for clinical

\section{Table 1. Search strategy}

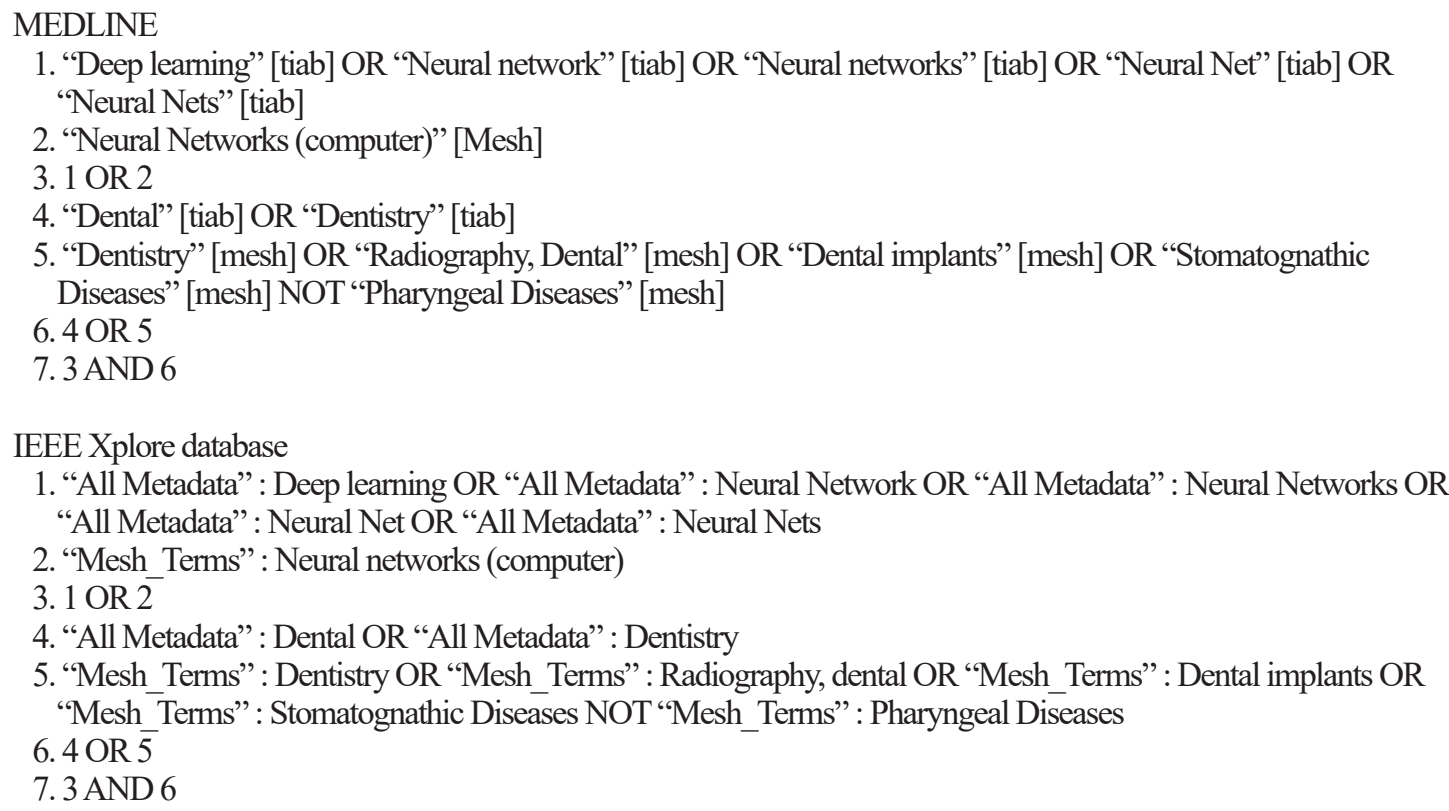


purpose rather than data mining or statistical analysis and (2) papers based on studies using deep neural networks such as convolution neural networks (CNNs), recurrent neural networks (RNNs), or generative adversarial networks (GANs), rather than machine learning among the AI fields.

\section{Data Extraction}

From the selected studies, we extracted information of the authors, publication years, deep learning architectures that were used, input data, output data, and performance metrics of the algorithm. We examine these data in detail below.

1) Deep learning architectures

(1) $\mathrm{CNNs}$

CNNs attracted attention after they won the ImageNet Challenge from 2012-2017, which is a largescale image recognition contest for classifying 50,000 high-resolution color images into 1,000 categories after training 1.2 million images, held every year since 2010 (Fig. 2). In 2012, AlexNet² decreased the top-5 error rate by $10 \%$ to $16.4 \%$, and SENet achieved $2.3 \%$ in 2017 .

The origin of the $\mathrm{CNN}$ is the Neocognitron Model, ${ }^{3}$ which applied a neurophysiological theory to an artificial neural network based on the principle that only certain neurons in the visual cortex are activated according to the shape of target object. ${ }^{4}$ CNNs largely comprise three layers: convolutional layer, pooling layer, and fully connected layer. The convolutional layer creates a feature map by arranging the outputs of convolution operation at each position of square filter while the filter is sliding over the input

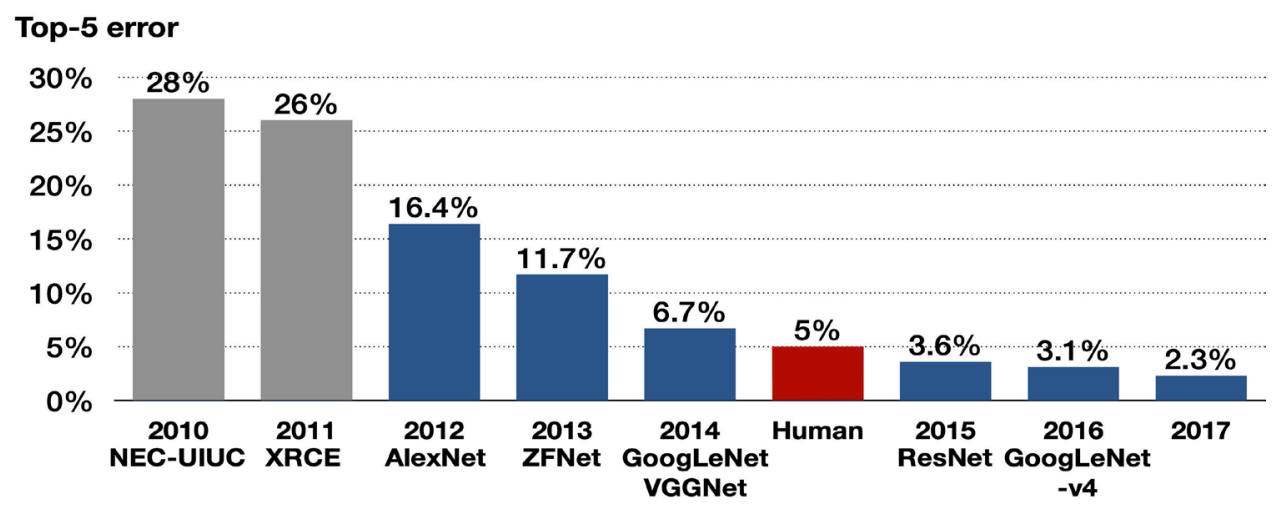

Fig. 2. Algorithms that won the ImageNet Large Scale Visual Recognition Challenge (ILSVRC) in 20102017. The top- 5 error refers to the probability that all top- 5 classifications proposed by the algorithm for the image are wrong. The algorithms with blue graph are convolutional neural network. Although VGGNet took second place in 2014, it is widely used in studies as its concise structure. Adapted from "A fully-automated deep learning pipeline for cervical cancer classification” by Alyafeai Z., Ghouti L., Expert Systems with Applications Proceedings of the IEEE 2019;141;112951. Copyright 2019 by Elsevier Ltd. 
data. It has the advantage of preserving horizontal and vertical information among pixels compared to the fully connected neural network, which converts images to one-dimensional vector. The pooling layer downsamples size of the feature map and summarizes important information in the feature map; the classification value is then output through the fully connected layer. For example, LeNet, which was the first $\mathrm{CNN}$ that classified hand-written numbers with an error rate of $0.95 \%$, comprises three convolutional layers, three pooling layers, and one fully connected layer (Fig. 3). ${ }^{5}$

Meanwhile, U-Net, which is used for region segmentation of medical images, does not have a fully connected layer. It comprises an encoder part, which extracts a feature map by convolution and pooling, and a decoder part, which restores the segmented images from the feature map by "up-convolution" (Fig. 4).

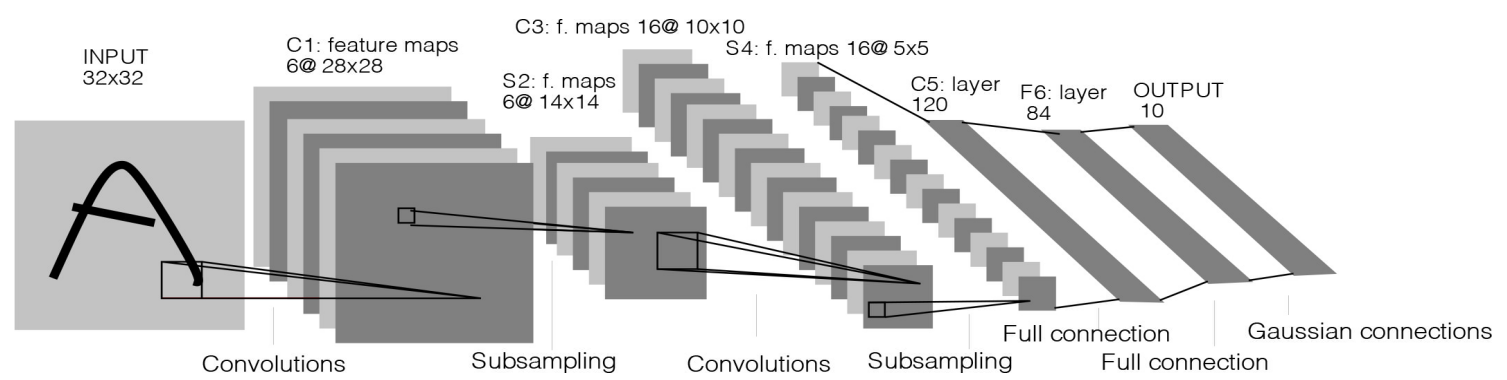

Fig. 3. Architecture of LeNet-5. Reprinted from "Gradient-based learning applied to document recognition” by LeCun Y., Bottou L, Bengio Y. et al., Proceedings of the IEEE 1998;86;2278-2323. Copyright 1998 by IEEE.

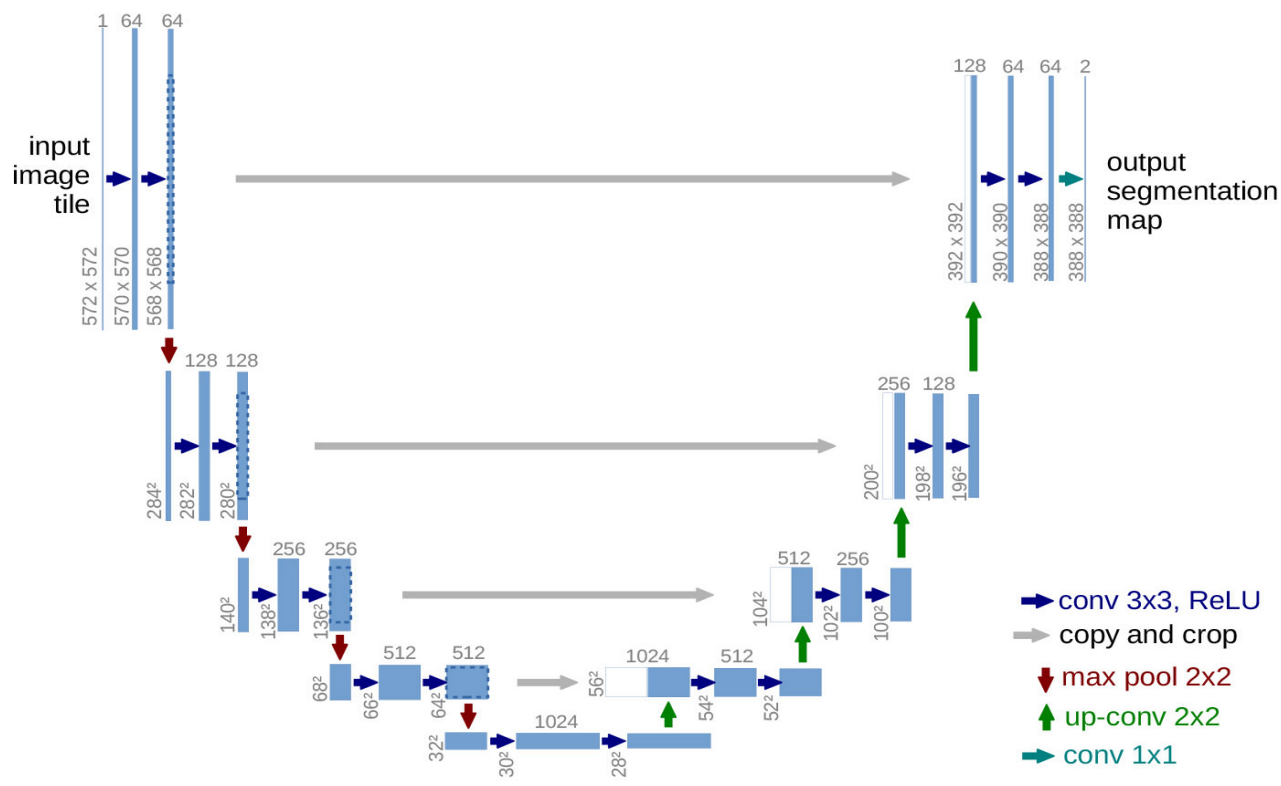

Fig. 4. Architecture of U-net. Reprinted from "U-net: Convolutional networks for biomedical image segmentation" by Ronneberger O., Fischer P., Bottou L, Brox T., Lecture Notes in Computer Science 2015;9351:234-241. Copyright 2015 by Elsevier Ltd. 
When detecting multiple objects in a single image, a region-based CNN (R-CNN) is used, which includes a region proposal network for the recognition of objects and their positions (Fig. 5). ${ }^{7}$ The region proposal network suggests anchor boxes of various ratios and sizes for the input image, and those that have a high intersection-over-union (IOU) with the previously trained images are selected.

(2) RNNs

RNNs can analyze time-series data that are arranged in chronological sequence such as voice signals. Therefore, they are utilized to predict indices such as stocks, for voice recognition, text translation, adding image captions, and image or music generation. A video of former US president Barack Obama appearing to give a speech has been published, in which the algorithm synthesize lip motion synchronized with his original voice. ${ }^{8}$ This neural network receives the input values from not only the previous layer $\left(X_{t}\right)$ but also the recurrent neurons of the previous time step, transforms, and delivers them to the next layer and recurrent neurons of the next time step, unlike the feed-forward neural network that only delivers signals from the input layer to the output layer (Fig. 6).

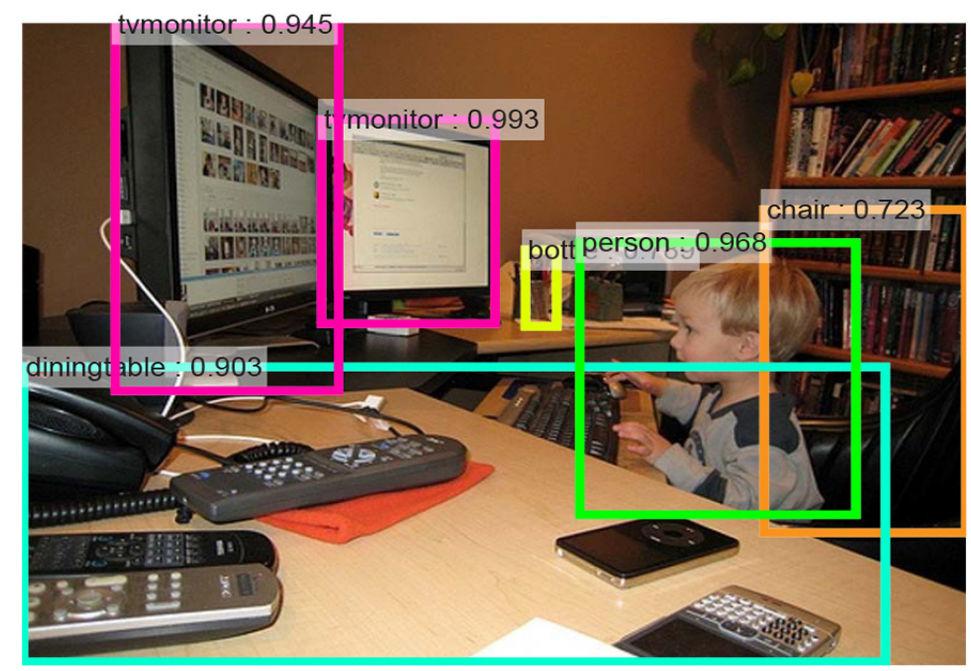

Fig. 5. Multiple object recognition in region-based convolutional neural network. Reprinted from "Faster R-CNN: Towards Real-Time Object Detection with Region Proposal Networks" by Ren S., He, K., Girshick, R., Sun, J., IEEE Transactions on Pattern Analysis and Machine intelligence 2017:39(6):11371149.

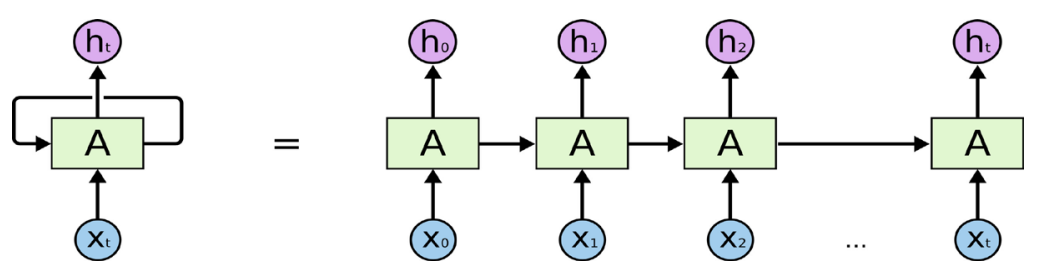

Fig. 6. Structure of recurrent neural network. Right illustrates unfold structure from left to right over time. Reprinted from http://colah.github.io/posts/2015-08-Understanding-LSTMs/ 
When an RNN-in a pure sense (also referred to as a "vanilla RNN")—-with the above characteristics is configured with deep layers, there are problems such as gradient vanishing/exploding and the longterm dependency. To solve these problems, changes in connections among cells (the units of neural networks) including skip connection or leaky units, long short-term memory cells, ${ }^{9}$ and gated recurrent unit cells ${ }^{10}$ using gates inside the cells have been proposed.

\section{(3) GANs}

GANs are unsupervised learning algorithms, ${ }^{11}$ which have a neural network generating an answer inside a neural network (the generator) competes with a neural network that evaluates it (the discriminator). The fake answers proposed by the generator are gradually similar to the ground truth with the aid of the feedback from the discriminator.

\section{2) Output data of deep learning}

The results of deep learning image analysis can be largely divided into five types as follows (Fig. 7). (1) Classification: The objects in image are classified as the most likely option to be ground truth among predetermined options. One example is LeNet-5, which classified hand-written numbers into 10 types, from 0 to 9. (2) Object localization: This is to indicate the locations of objects in image by bounding boxes. When object localization and classification are performed simultaneously, it is called object detection. (3) Semantic segmentation: This means to segment whole image according to the pixel-based classification without object recognition. (4) Instance segmentation: This recognizes each object and delineates its outline in an image. (5) Image reconstruction: Examples include image quality enhancement by super-resolution or artifact reduction, and class activation maps overlap heat map, which changes the color depending on the contribution of the classification, to the input image. This allows visual confirmation based on which areas of the image are classified using the deep learning algorithm.
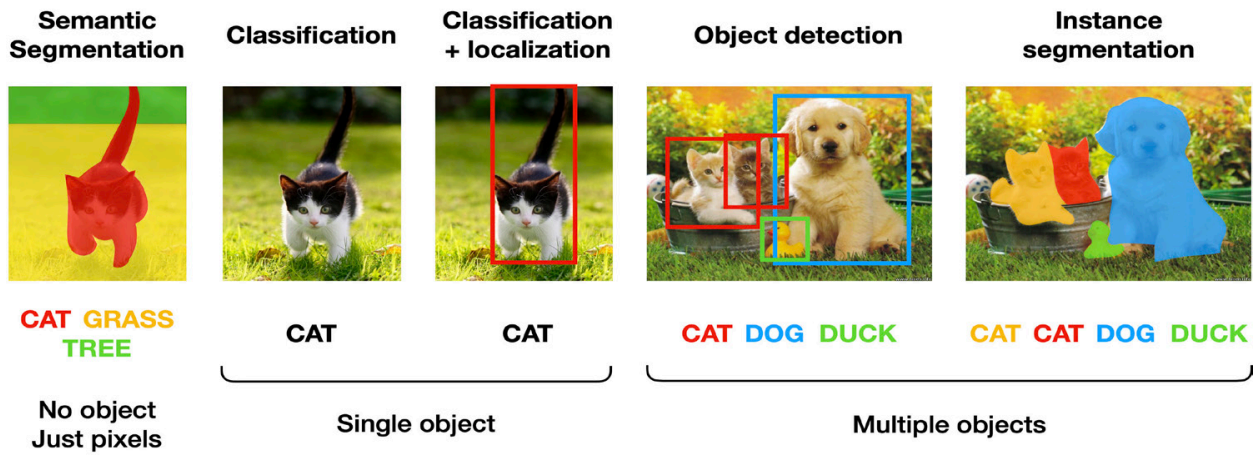

Fig. 7. Computer vision tasks. Adapted from "https://www.slideshare.net/darian_f/introduction-to-theartificial-intelligence-and-computer-vision-revolution." 
3) Performance metrics of deep learning algorithms

The representative performance metrics for classification algorithms are accuracy, precision, recall, F1 score, and the area under the receiver operating characteristic curve (AUC). Other metrics except AUC can be calculated using the confusion matrix illustrating whether the predicted classification matches the ground truth (Fig. 8).

For example, when we evaluate the accuracy of a deep learning model that classifies images into three types, we can calculate the accuracy simply by dividing the number of cases which classify A as A, B as $\mathrm{B}$, or $\mathrm{C}$ as $\mathrm{C}$ by the total number of cases.

$$
\text { Accuracy }=\frac{T P_{A}+T P_{B}+T P_{C}}{\text { Total }}
$$

( $T P=$ True Positive, $F P=$ False Positive, $T N=$ True Negative, $F N=$ False Negative)

Furthermore, the $\mathrm{F}_{1}$ score can be calculated by determining the precisions $\left(\right.$ Precision $_{A}$, Precision $_{B}$, and Precision $\left._{C}\right)$ and recalls $\left(\right.$ Recall $_{A}, \operatorname{Recall}_{B}$, and Recall $C$ ) for classifying A, B, and C, and calculating the mean precision $\left(\right.$ Precision $\left._{\text {mean }}\right)$ and mean recall $\left(\right.$ Recall $\left._{\text {mean }}\right)$, and then calculating the harmonic mean of these two.

$$
\begin{gathered}
\text { Precision }_{A}=\frac{T P_{A}}{T P_{A}+F P_{A}}, \quad \operatorname{Recall}_{A}=\frac{T P_{A}}{T P_{A}+F N_{A},} \\
\left.F_{1} \text { score }=\frac{2}{\frac{1}{\text { Precisionmean }_{\text {meall }}+\frac{1}{\text { Rean }}}} \text { (harmonic mean }\right)
\end{gathered}
$$

The evaluation indices for object localization and segmentation include the IOU and the dice similarity coefficient, in addition to the above-mentioned indices (Fig. 9). IOU is also called Jaccard index and is calculated by dividing the overlapping area between the ground truth and the predicted areas by the union area. The dice similarity coefficient is calculated by dividing the double of the overlapping area by the sum of each area.
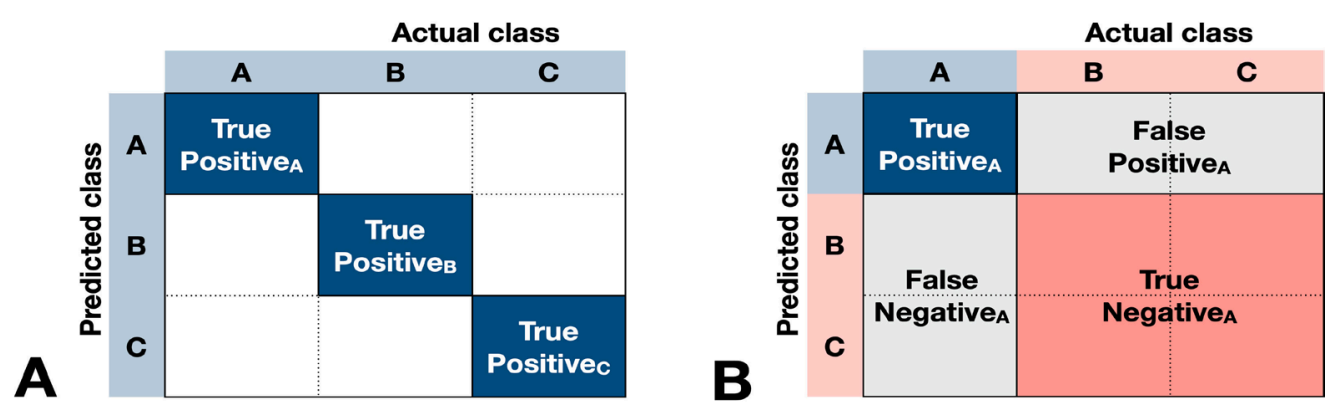

Fig. 8. Confusion matrix to calculate accuracy (A) and to calculate precision recall, and F1 score (B). 


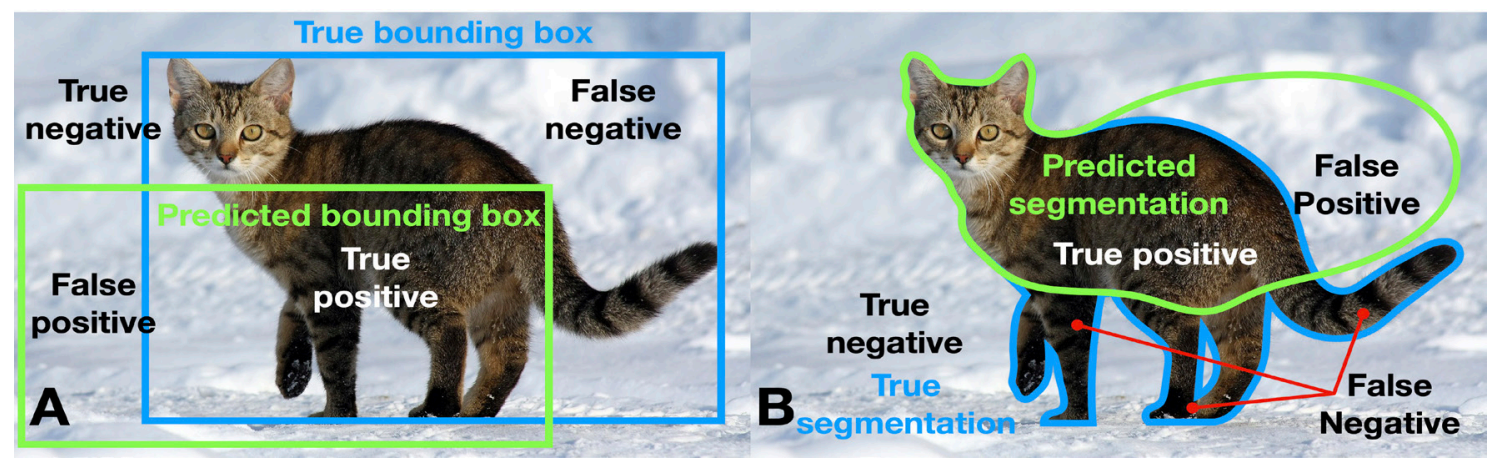

Fig. 9. Evaluation of object localization (A) and object segmentation (B).

$$
I O U=\frac{|A \cap B|}{|A \cup B|}=\frac{T P}{T P+F P+F N} \quad D S C=\frac{2|A \cap B|}{|A|+|B|}=\frac{2 T P}{2 T P+F P+F N}
$$

\section{III . Results}

\section{Literature Search and Selection of Studies}

We found 340 papers by searching MEDLINE and the IEEE Xplore Library, excluding 7 duplicates. After evaluating the titles and abstracts, we excluded 272 papers and evaluated the full texts of 68 papers. A total of 62 papers were included in the study (Fig. 10). The excluded papers and the reasons for their exclusion are outlined in Suppl. 1.

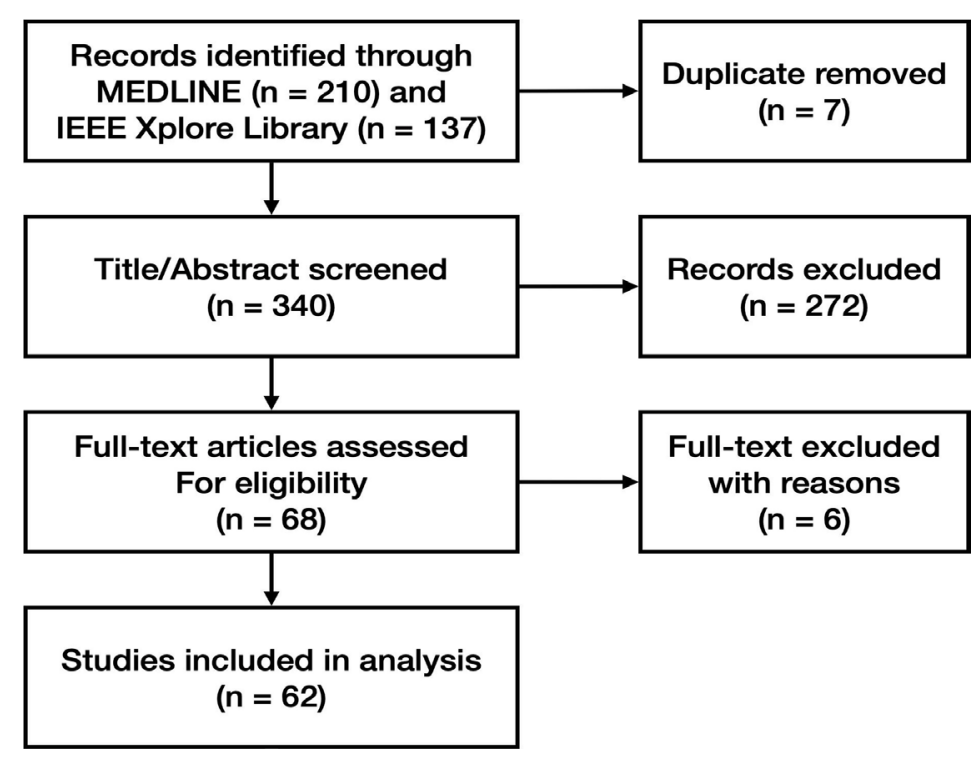

Fig. 10. Flow chart showing literature search and selection. 


\section{Data Extraction}

The characteristics of the selected studies and the extracted data are listed in Table 2.

\section{Table 2. Characteristics of included studies}

\begin{tabular}{|c|c|c|c|c|}
\hline $\begin{array}{l}\text { Author } \\
\text { Year }\end{array}$ & Architecture & Input & Output & Performance metrics \\
\hline \multicolumn{5}{|c|}{$\begin{array}{l}\text { 1. Detection and segmentation of tooth and oral anatomy } \\
1.1 \text { Tooth localization and numbering }\end{array}$} \\
\hline $\begin{array}{l}\text { Eun } \\
2016^{74}\end{array}$ & $\begin{array}{l}\text { Sliding window } \\
\text { technique with } \\
\text { CNN }\end{array}$ & $\begin{array}{l}7,662 \text { backgrounds, } \\
651 \text { single-root teeth, } \\
\text { and } 484 \text { multi-root } \\
\text { teeth images cropped } \\
\text { from } 500 \text { periapical } \\
\text { radiographs }\end{array}$ & $\begin{array}{l}\text { Multiple object } \\
\text { localization(tooth) }\end{array}$ & $\begin{array}{l}\text { Mean average best } \\
\text { overlap }=0.714\end{array}$ \\
\hline $\begin{array}{l}\text { Oktay } \\
2017^{75}\end{array}$ & $\begin{array}{l}\text { Sliding window } \\
\text { technique with } \\
\text { CNN } \\
\text { (AlexNet) }\end{array}$ & $\begin{array}{l}100 \text { panoramic } \\
\text { radiographs }\end{array}$ & $\begin{array}{l}\text { Classification } \\
\text { (anterior, premolar, } \\
\text { molar) }\end{array}$ & $\begin{array}{l}\text { Accuracy }(\text { anterior })=0.9247, \\
\text { Accuracy }(\text { premolar })=0.9174, \\
\text { Accuracy }(\text { molar })=0.9432\end{array}$ \\
\hline $\begin{array}{l}\text { Miki } \\
2017^{76}\end{array}$ & $\begin{array}{l}\text { CNN } \\
\text { (AlexNet) }\end{array}$ & $\begin{array}{l}\text { Cropped CBCT slices } \\
\text { from } 52 \text { participants } \\
(227 \times 227 \text { pixel })\end{array}$ & $\begin{array}{l}\text { Classification } \\
\text { (central incisor, } \\
\text { lateral incisor, canine, } \\
1^{\text {st }} \text { premolar, } 2^{\text {nd }} \\
\text { premolar, } 1^{\text {st }} \text { molar, } \\
2^{\text {nd }} \text { molar) }\end{array}$ & $\begin{array}{l}\text { Dataset } \mathrm{D} \text { (rotation \& gamma } \\
\text { correction) } \\
\text { Accuracy }=0.888\end{array}$ \\
\hline \multirow[t]{2}{*}{$\begin{array}{l}\text { Zhang } \\
2018^{14}\end{array}$} & \multirow{2}{*}{$\begin{array}{l}\text { Cascade network } \\
=3 \\
\text { CNNs(ImageNet } \\
\text { pre-trained } \\
\text { VGG16) + } 1 \text { logic } \\
\text { refine module }\end{array}$} & \multirow[t]{2}{*}{$\begin{array}{l}1,000 \text { periapical } \\
\text { radiographs }\end{array}$} & $\begin{array}{l}\text { Multiple object } \\
\text { localization(tooth) }\end{array}$ & $\begin{array}{l}\text { Precision }=0.980, \\
\text { Recall }=0.983, \\
\text { F1 score }=0.981\end{array}$ \\
\hline & & & $\begin{array}{l}\text { Classification } \\
\text { (tooth numbering) }\end{array}$ & $\begin{array}{l}\text { Precision }=0.958, \\
\text { Recall }=0.961, \\
\text { F1 score }=0.959\end{array}$ \\
\hline \multirow[t]{2}{*}{$\begin{array}{l}\text { Chen } \\
2019^{12}\end{array}$} & \multirow[t]{2}{*}{$\begin{array}{l}\text { Faster } \\
\text { R-CNN with } \\
\text { inception ResNet v2 }\end{array}$} & \multirow{2}{*}{$\begin{array}{l}1,250 \text { periapical } \\
\text { radiographs } \\
{[(300-500) \times(300-4} \\
00] \text { pixel })\end{array}$} & $\begin{array}{l}\text { Multiple object } \\
\text { localization(tooth) }\end{array}$ & $\begin{array}{l}\text { Precision }=0.900, \\
\text { Recall }=0.985 \\
\text { IOU }=0.91\end{array}$ \\
\hline & & & $\begin{array}{l}\text { Classification } \\
\text { (tooth numbering) }\end{array}$ & $\begin{array}{l}\text { Precision }=0.715, \\
\text { Recall }=0.782\end{array}$ \\
\hline
\end{tabular}




\begin{tabular}{|c|c|c|c|c|}
\hline \multirow[t]{4}{*}{$\begin{array}{l}\text { Tuzoff } \\
2019^{13}\end{array}$} & \multirow[t]{2}{*}{$\begin{array}{l}\text { Faster R-CNN with } \\
\text { VGG16 }\end{array}$} & \multirow[t]{4}{*}{$\begin{array}{l}1,574 \text { panoramic } \\
\text { radiographs }\end{array}$} & $\begin{array}{l}\text { Multiple object } \\
\text { localization(tooth) }\end{array}$ & $\begin{array}{l}\text { Precision }=0.9945, \\
\text { Recall }^{\dagger}=0.9941\end{array}$ \\
\hline & & & $\begin{array}{l}\text { Classification } \\
\text { (tooth numbering) }\end{array}$ & $\begin{array}{l}\text { Specificity }=0.9994, \\
\text { Recall }^{\dagger}=0.9800\end{array}$ \\
\hline & \multirow[t]{2}{*}{ Expert } & & $\begin{array}{l}\text { Multiple object } \\
\text { localization(tooth) }\end{array}$ & $\begin{array}{l}\text { Precision }=0.9998, \\
\text { Recall }^{\dagger}=0.9980\end{array}$ \\
\hline & & & $\begin{array}{l}\text { Classification } \\
\text { (tooth numbering) }\end{array}$ & $\begin{array}{l}\text { Specificity }=0.9997, \\
\text { Recall }{ }^{\dagger}=0.9893\end{array}$ \\
\hline \multirow[t]{2}{*}{$\begin{array}{l}\text { Koch } \\
2019^{77}\end{array}$} & $\begin{array}{l}6 \text { modifications of } \\
\text { U-Net }\end{array}$ & \multirow[t]{2}{*}{$\begin{array}{l}1,500 \text { panoramic } \\
\text { radiographs }\end{array}$} & \multirow{2}{*}{$\begin{array}{l}\text { Classification }(1-4 \text { : } \\
32, \text { teeth with/without } \\
\text { restoration and } \\
\text { with/without } \\
\text { orthodontic } \\
\text { appliance, } 5: \text { implant, } \\
6:>32 \text { teeth , } 7-10 \text { : } \\
<32 \text { teeth } \\
\text { with/without } \\
\text { restoration and } \\
\text { with/without } \\
\text { orthodontic appliance) }\end{array}$} & $\begin{array}{l}\text { Ensemble of } \mathrm{U}-\mathrm{Net} \\
\text { modification } 1 \text { and } 4 \\
\text { Accuracy }=0.952, \\
\text { Precision }=0.933, \\
\text { Recall }{ }^{\dagger}=0.944, \\
\text { Specificity }=0.961, \\
\text { DSC }=0.936\end{array}$ \\
\hline & Mask R-CNN & & & $\begin{array}{l}\text { Accuracy }=0.98, \\
\text { Precision }=0.94, \\
\text { Recall }{ }^{\dagger}=0.84, \\
\text { Specificity }=0.99, \text { DSC }=0.88\end{array}$ \\
\hline \multirow[t]{3}{*}{$\begin{array}{l}\text { Hiraiwa } \\
2019^{78}\end{array}$} & CNN(AlexNet) & \multirow{3}{*}{$\begin{array}{l}760 \text { cropped images } \\
\text { of mandibular } 1^{\text {st }} \\
\text { molar from } 400 \\
\text { panoramic } \\
\text { radiographs } \\
\text { CBCT images from } \\
400 \text { participants } \\
\text { (ground truth) }\end{array}$} & \multirow[t]{3}{*}{$\begin{array}{l}\text { Prediction(number of } \\
\text { distal root of } \\
\text { mandibular } 1^{\text {st }} \text { molar) }\end{array}$} & $\begin{array}{l}\text { Accuracy }=0.874, \\
\text { Recall }{ }^{\dagger}=0.773, \\
\text { Specificity }=0.971, \\
\text { Precision }{ }^{\dagger}=0.963, \\
\text { NPV }=0.818, \text { AUC }=0.87, \\
\text { Training time }=51 \text { minutes, } \\
\text { Testing time }=9 \text { seconds }\end{array}$ \\
\hline & CNN(GoogleNet) & & & $\begin{array}{l}\text { Accuracy }=0.853, \\
\text { Recall }{ }^{\dagger}=0.742, \\
\text { Specificity }=0.959, \\
\text { Precision }{ }^{\dagger}=0.947, \\
\text { NPV }=0.800, \text { AUC }=0.85, \\
\text { Training time }=3 \text { hours, } \\
\text { Testing time }=11 \text { seconds }\end{array}$ \\
\hline & Expert radiologist & & & $\begin{array}{l}\text { Accuracy }=0.812, \\
\text { Recall }{ }^{\dagger}=0.802, \\
\text { Specificity }=0.820 \\
\text { Precision }{ }^{\dagger}=0.787, \\
\mathrm{NPV}=0.834, \text { AUC }=0.74\end{array}$ \\
\hline \multicolumn{5}{|c|}{ 1.2. Tooth segmentation } \\
\hline \multirow[t]{2}{*}{$\begin{array}{l}\text { Jader } \\
2018^{15}\end{array}$} & \multirow[t]{2}{*}{$\begin{array}{l}\text { Mask R-CNN } \\
\text { with ResNet101 }\end{array}$} & \multirow[t]{2}{*}{$\begin{array}{l}1,500 \text { panoramic } \\
\text { radiographs }\end{array}$} & $\begin{array}{l}\text { Instance } \\
\text { segmentation }\end{array}$ & None \\
\hline & & & $\begin{array}{l}\text { Classification }(1-4: \\
32 \text {, teeth with/without } \\
\text { restoration and } \\
\text { with/without } \\
\text { orthodontic } \\
\text { appliance, } 5: \text { implant, } \\
6:>32 \text { teeth , } 7-10 \text { : } \\
<32 \text { teeth } \\
\text { with/without } \\
\text { restoration and } \\
\text { with/without } \\
\text { orthodontic } \\
\text { appliance) }\end{array}$ & $\begin{array}{l}\text { Accuracy }=0.98, \\
\text { Precision }=0.94, \\
\text { Recall }=0.84, \\
\text { F1 score }=0.88, \\
\text { Specificity }=0.99\end{array}$ \\
\hline
\end{tabular}




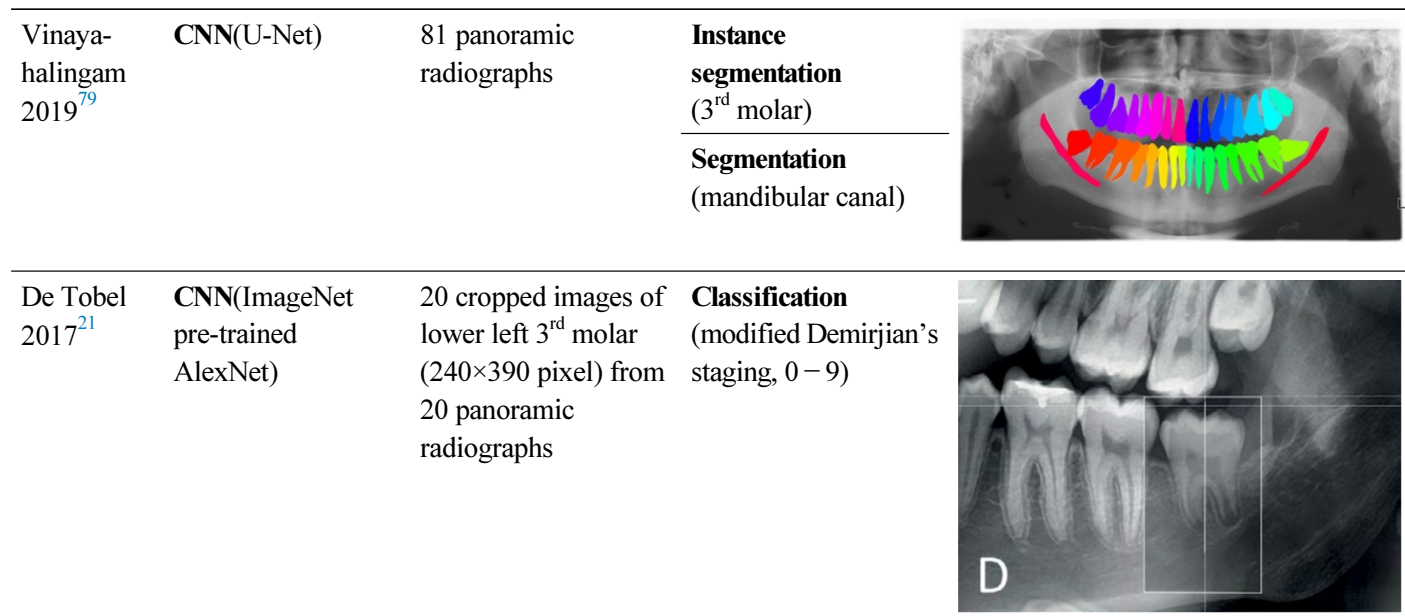

\begin{tabular}{|c|c|c|c|c|}
\hline \multirow[t]{2}{*}{$\begin{array}{l}\text { Merdietio } \\
2019^{16}\end{array}$} & \multirow[t]{2}{*}{ CNN(AlexNet) } & \multirow[t]{2}{*}{$\begin{array}{l}400 \text { panoramic } \\
\text { radiographs }\end{array}$} & $\begin{array}{l}\text { Segmentation } \\
\text { (lower left } 3^{\text {rd }} \text { molar) }\end{array}$ & Accuracy $=0.61$ \\
\hline & & & $\begin{array}{l}\text { Classification } \\
\text { (Modified } \\
\text { Demirjian's staging, } \\
0-9 \text { ) }\end{array}$ & $\begin{array}{l}\text { Accuracy }=0.61 \\
\text { Mean absolute } \\
\text { difference }=0.53 \text { stages } \\
\text { Cohen's } \kappa \text { linear }=0.84\end{array}$ \\
\hline \multirow[t]{3}{*}{$\begin{array}{l}\text { Tian } \\
2019^{17}\end{array}$} & \multirow{2}{*}{$\begin{array}{l}\text { CNN(sparse octree } \\
\text { structure, } \\
\text { voxel-based) }\end{array}$} & \multirow[t]{3}{*}{$\begin{array}{l}\text { 3D scanned images of } \\
600 \text { dental models }\end{array}$} & $\begin{array}{l}\text { Classification } \\
\text { (tooth numbering) }\end{array}$ & $\begin{array}{l}\text { Recall }^{\dagger}=0.9800, \\
\text { Specificity }=0.9994\end{array}$ \\
\hline & & & $\begin{array}{l}\text { Instance } \\
\text { segmentation(tooth) }\end{array}$ & Accuracy $=0.8981$ \\
\hline & Expert & & $\begin{array}{l}\text { Classification } \\
\text { (tooth numbering) }\end{array}$ & $\begin{array}{l}\text { Recall }^{\dagger}=0.9893 \\
\text { Specificity }=0.9997\end{array}$ \\
\hline $\begin{array}{l}\mathrm{Xu} \\
2019^{18}\end{array}$ & CNN & $\begin{array}{l}\text { 3D scanned mesh } \\
\text { images from } 1,200 \\
\text { dental models }\end{array}$ & $\begin{array}{l}\text { Instance } \\
\text { segmentation } \\
\text { (tooth - gingiva, } \\
\text { tooth - tooth) }\end{array}$ & $\begin{array}{l}\text { Accuracy }(\text { maxilla })=0.9906 \\
\text { Accuracy }(\text { mandible })=0.9879\end{array}$ \\
\hline
\end{tabular}

\begin{tabular}{|c|c|c|c|c|}
\hline \multicolumn{5}{|c|}{ 1.3. Bone segmentation } \\
\hline $\begin{array}{l}\text { Duong } \\
2019^{20}\end{array}$ & CNN(U-Net) & $\begin{array}{l}50 \text { intraoral ultrasonic } \\
\text { images on } 8 \text { lower } \\
\text { incisors from piglets } \\
(128 \times 128 \text { pixel })\end{array}$ & $\begin{array}{l}\text { Segmentation } \\
\text { (alveolar bone) }\end{array}$ & $\begin{array}{l}\mathrm{DSC}=75.0 \pm 12.7 \%, \\
\text { Recall }{ }^{\dagger}=77.8 \pm 13.2 \%, \\
\text { Specificity }=99.4 \pm 0.8 \%\end{array}$ \\
\hline
\end{tabular}




\begin{tabular}{|c|c|c|c|c|c|}
\hline \multirow[t]{2}{*}{$\begin{array}{l}\text { Minnema } \\
2019^{19}\end{array}$} & CNN(MS-D Net) & $\begin{array}{l}\text { CBCT from } 20 \\
\text { patients }\end{array}$ & \multicolumn{3}{|c|}{ Segmentation(bone) } \\
\hline & CNN(U-Net) & MS-D network & U-Net & ResNet & Snake evolution \\
\hline
\end{tabular}

Snake evolution

algorithm

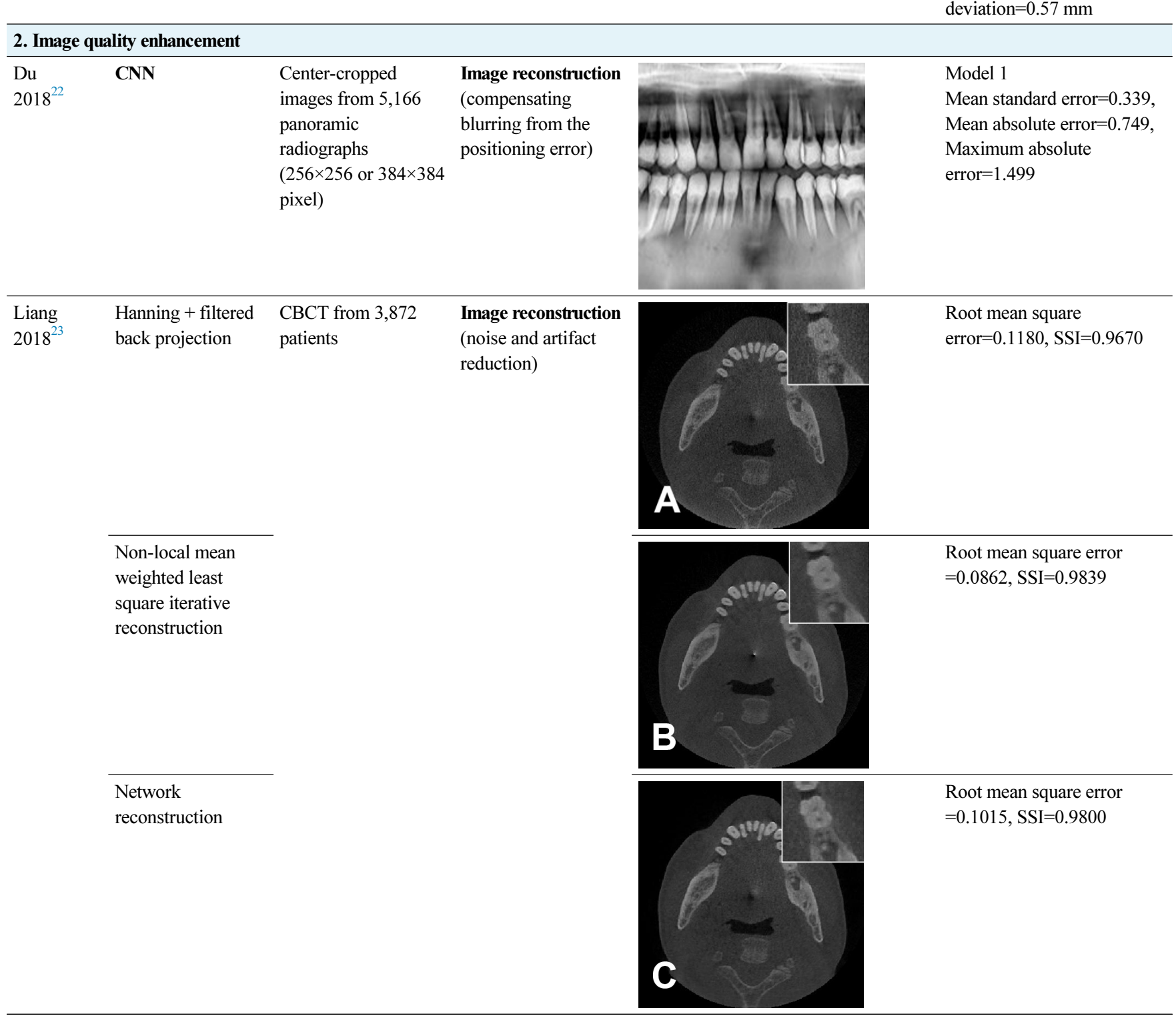




\begin{tabular}{|c|c|c|c|c|}
\hline \multirow[t]{3}{*}{$\begin{array}{l}\mathrm{Hu} \\
2019^{24}\end{array}$} & GAN & $\begin{array}{l}\text { Low-dose CBCT } \\
\text { images from } 44 \\
\text { patients }\end{array}$ & $\begin{array}{l}\text { Image reconstruction } \\
\text { (noise and artifact } \\
\text { reduction) }\end{array}$ & $\begin{array}{l}\operatorname{PSNR}\left(360^{\circ}\right)=32.657, \\
\operatorname{SSI}\left(360^{\circ}\right)=0.925, \\
\text { Noise suppression }=5.52 \pm 0.25 \text {, } \\
\text { Artifact correction }=6.98 \pm 0.35 \text {, } \\
\text { Detail restoration }=5.56 \pm 0.31 \text {, } \\
\text { Comprehensive } \\
\text { quality }=6.52 \pm 0.34, \\
\text { Training time per } \\
\text { batch }=0.691 \text {, } \\
\text { Testing time per batch }=0.183\end{array}$ \\
\hline & CNN & $\begin{array}{l}\text { (180 } 180^{\circ} \text { scanned } \\
\text { images, } 120360^{\circ} \\
\text { scanned images })\end{array}$ & & $\begin{array}{l}\operatorname{PSNR}\left(360^{\circ}\right)=34.402, \\
\operatorname{SSI}\left(360^{\circ}\right)=0.934, \\
\text { Noise suppression }=8.95 \pm 0.36 \text {, } \\
\text { Artifact correction }=7.20 \pm 0.23 \text {, } \\
\text { Detail restoration }=5.35 \pm 0.28 \text {, } \\
\text { Comprehensive } \\
\text { quality }=7.54 \pm 0.32, \\
\text { Training time per } \\
\text { batch }=0.726, \\
\text { Testing time per batch }=0.183\end{array}$ \\
\hline & m-WGAN & $\begin{array}{l}\text { Normal dose CBCT } \\
\text { images } \\
\text { (ground truth) }\end{array}$ & & $\begin{array}{l}\operatorname{PSNR}\left(360^{\circ}\right)=33.824, \\
\operatorname{SSI}\left(360^{\circ}\right)=0.975, \\
\text { Noise suppression }=8.20 \pm 0.35 \text {, } \\
\text { Artifact correction }=7.46 \pm 0.27 \text {, } \\
\text { Detail restoration }=8.98 \pm 0.20 \text {, } \\
\text { Comprehensive } \\
\text { quality }=8.25 \pm 0.21, \\
\text { Training time per } \\
\text { batch }=0.798, \\
\text { Testing time per batch }=0.184\end{array}$ \\
\hline \multirow[t]{4}{*}{$\begin{array}{l}\text { Hegazy } \\
2019^{25}\end{array}$} & \multirow[t]{2}{*}{ CNN(U-Net) } & \multirow{2}{*}{$\begin{array}{l}1,000 \text { projection } \\
\text { images }\left(0-180^{\circ}\right) \\
\text { from } 5 \text { patients who } \\
\text { had different kinds of } \\
\text { metal implants and } \\
\text { dental fillings at } \\
\text { different tooth } \\
\text { positions }\end{array}$} & $\begin{array}{l}\text { Segmentation } \\
\text { (metal) }\end{array}$ & $\begin{array}{l}\text { Mean } \mathrm{IOU}=0.94 \\
\text { Mean DSC }=0.96\end{array}$ \\
\hline & & & $\begin{array}{l}\text { Image reconstruction } \\
\text { (metal artifact } \\
\text { reduction) }\end{array}$ & $\begin{array}{l}\text { Mean relative error }=94.25 \% \\
\text { Mean normalized absolute } \\
\text { difference }=93.25 \% \\
\text { Mean sum of square } \\
\text { difference }=91.83 \%\end{array}$ \\
\hline & \multirow{2}{*}{$\begin{array}{l}\text { Conventional } \\
\text { segmentation } \\
\text { method }\end{array}$} & $\begin{array}{l}\text { Original CBCT } \\
\text { images }\end{array}$ & $\begin{array}{l}\text { Segmentation } \\
\text { (metal) }\end{array}$ & $\begin{array}{l}\text { Mean } \mathrm{IOU}=0.75 \\
\text { Mean } \mathrm{DSC}=0.86\end{array}$ \\
\hline & & $+\infty$ & $\begin{array}{l}\text { Image reconstruction } \\
\text { (metal artifact } \\
\text { reduction) }\end{array}$ & $\begin{array}{l}\text { Mean relative error }=91.71 \% \\
\text { Mean normalized absolute } \\
\text { difference }=95.06 \% \\
\text { Mean sum of square } \\
\text { difference }=93.64 \%\end{array}$ \\
\hline
\end{tabular}




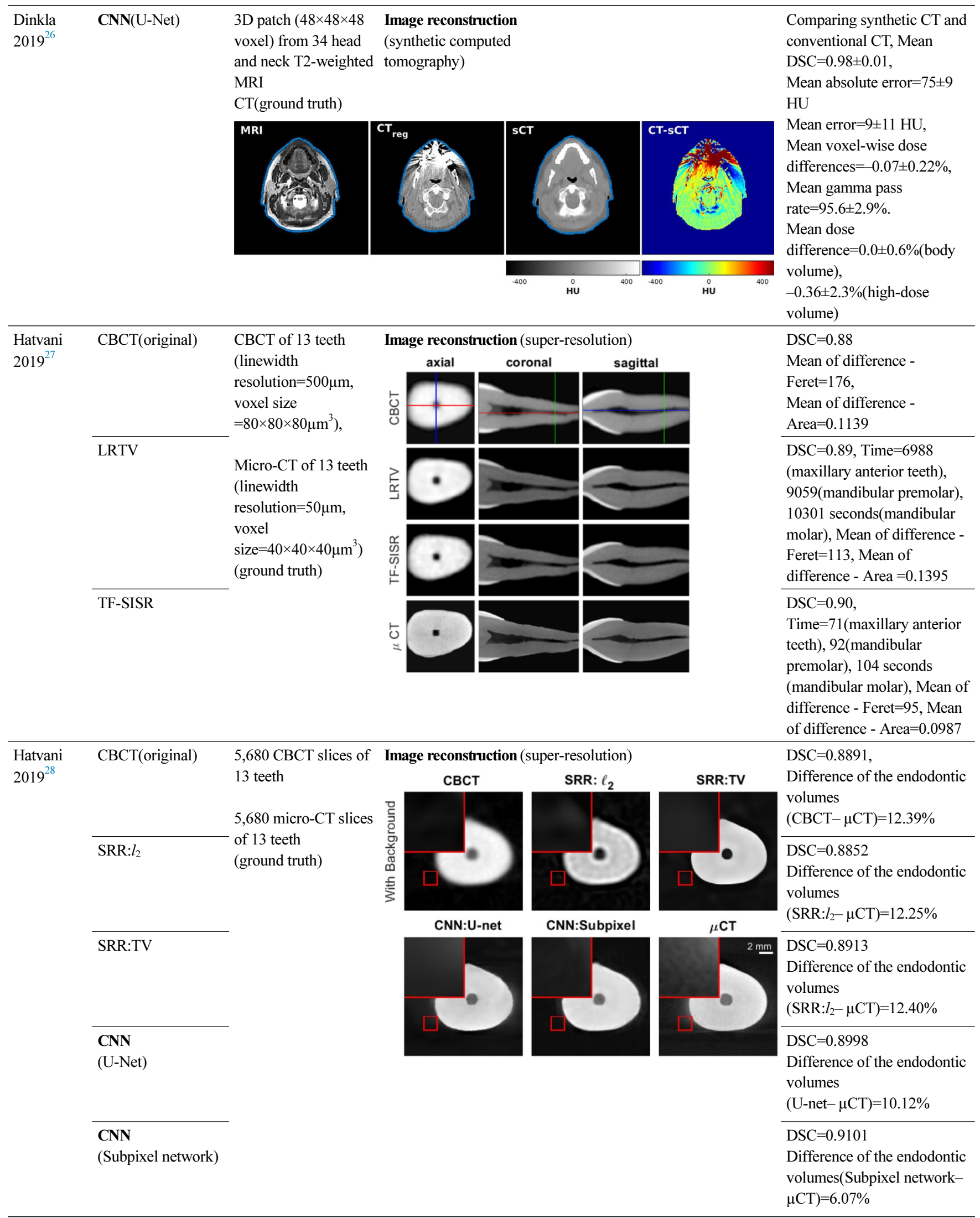




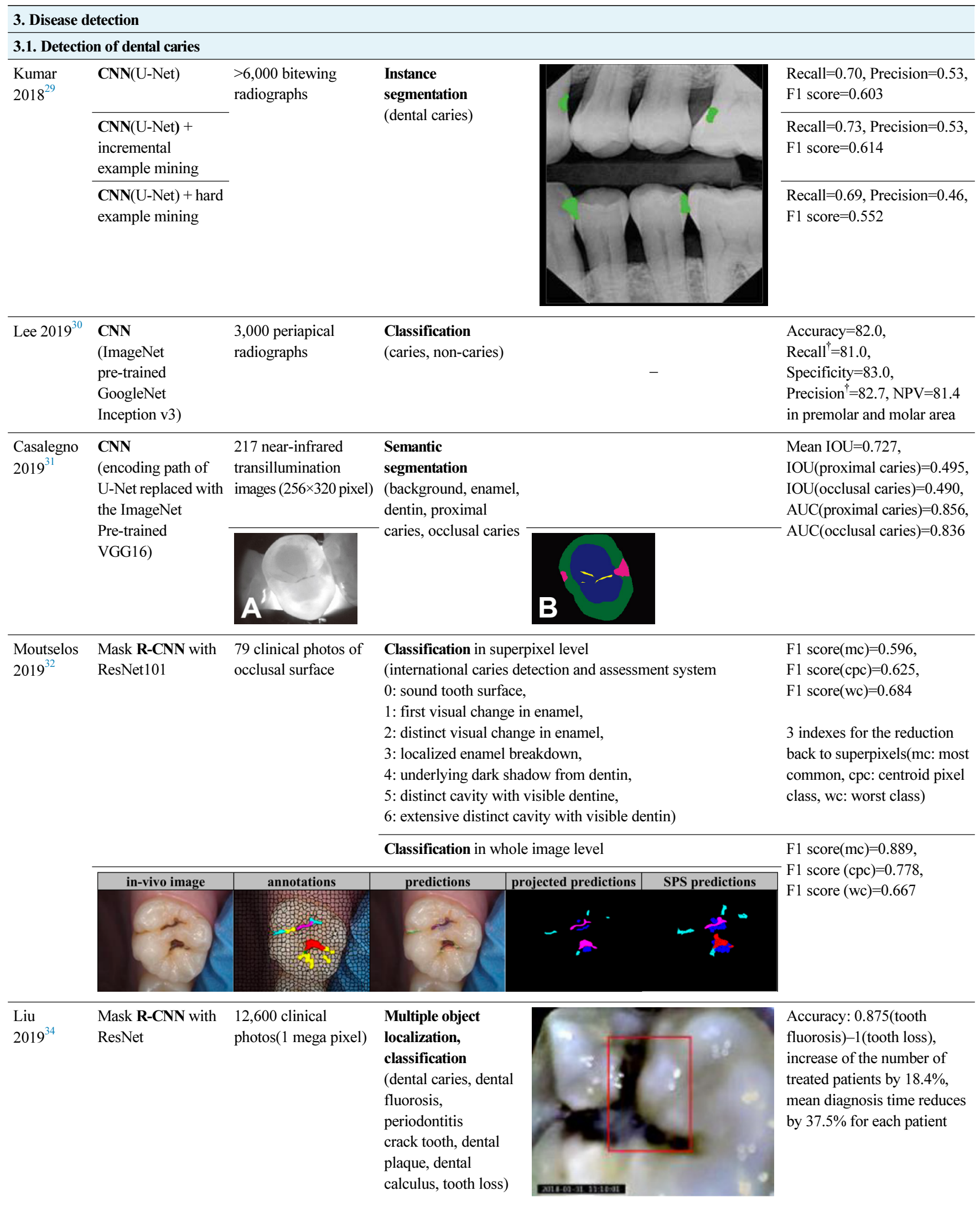




\begin{tabular}{|c|c|c|c|c|}
\hline \multicolumn{5}{|c|}{ 3.2. Detection of dental plaque and periodontal disease } \\
\hline $\begin{array}{l}\text { Yauney } \\
2017^{80}\end{array}$ & $\begin{array}{l}\text { CNN } \\
\text { (truncated version } \\
\text { of VGG16) }\end{array}$ & $\begin{array}{l}\text { A } \\
47(\mathrm{CD} \text { database }) \text { and } \\
49(\mathrm{RD} \text { database }) \text { pairs } \\
\text { of white light mode } \\
\mathrm{B} \\
\text { and plaque mode } \\
\text { intraoral photo } \\
(512 \times 384 \text { pixel })\end{array}$ & $\begin{array}{l}\text { Semantic } \\
\text { segmentation } \\
\text { (plaque, non-plaque) }\end{array}$ & $\begin{array}{l}\text { Accuracy }=0.8718, \\
\text { AUC }=0.8720\end{array}$ \\
\hline $\begin{array}{l}\text { Bezruk } \\
2017^{35}\end{array}$ & CNN & $\begin{array}{l}\text { Malondialdehyde } \\
\text { concentration, } \\
\text { Gluthatione } \\
\text { concentration, } \\
\text { Sulcus bleeding index }\end{array}$ & $\begin{array}{l}\text { Classification } \\
\text { (normal, gingivitis) }\end{array}$ & $\begin{array}{l}\text { Precision }=0.80, \\
\text { Recall }=0.78, \\
\text { F1 score }=0.78\end{array}$ \\
\hline $\begin{array}{l}\text { Aberin } \\
2018^{36}\end{array}$ & CNN(AlexNet) & $\begin{array}{l}1,000 \text { grayscale } \\
\text { images with } 600 \\
\text { magnification } \\
(227 \times 227 \text { pixel })\end{array}$ & $\begin{array}{l}\text { Classification } \\
\text { (healthy, unhealthy) }\end{array}$ & $\begin{array}{l}\text { Accuracy }=0.76, \\
\text { Mean square error }=0.05, \\
\text { Precision }=0.68, \\
\text { Recall }=0.98\end{array}$ \\
\hline $\begin{array}{l}\text { Joo } \\
2019^{37}\end{array}$ & CNN & $\begin{array}{l}1,843 \text { clinical photos } \\
\text { of periodontal tissue }\end{array}$ & $\begin{array}{l}\text { Classification } \\
\text { (healthy periodontal } \\
\text { status, mild } \\
\text { periodontitis, severe } \\
\text { periodontitis, not } \\
\text { periodontal image) }\end{array}$ & $\begin{array}{l}\text { Accuracy }(\text { healthy })=0.83, \\
\text { Accuracy }(\text { mild } \\
\text { periodontitis })=0.74, \\
\text { Accuracy }(\text { severe } \\
\text { periodontitis })=0.70, \\
\text { Accuracy(not periodontal } \\
\text { image })=0.94\end{array}$ \\
\hline \multirow[t]{2}{*}{$\begin{array}{l}\text { Krois } \\
2019^{38}\end{array}$} & CNN & \multirow[t]{2}{*}{$\begin{array}{l}2,001 \text { cropped } \\
\text { panoramic } \\
\text { radiographs }\end{array}$} & \multirow[t]{2}{*}{$\begin{array}{l}\text { Classification } \\
(<20 \% \text { bone loss, } \\
\geq 20 \% \text { bone loss })\end{array}$} & $\begin{array}{l}\text { Accuracy }=0.81, \\
\text { Precision }{ }^{\dagger}=0.76, \\
\text { Recall }^{\dagger}=0.81, \\
\text { Specificity }=0.81, \mathrm{NPV}=0.85, \\
\text { AUC }=0.89, \text { F1 score }=0.78\end{array}$ \\
\hline & Dentist & & & $\begin{array}{l}\text { Accuracy }=0.76, \\
\text { Precision }^{\dagger}=0.68, \\
\text { Recall }^{\dagger}=0.92, \\
\text { Specificity }=0.63, \mathrm{NPV}=0.90, \\
\text { AUC }=0.77, \mathrm{~F} 1 \text { score }=0.78\end{array}$ \\
\hline \multicolumn{5}{|c|}{ 3.3. Detection of periapical diseases } \\
\hline $\begin{array}{l}\text { Prajapati } \\
2017^{33}\end{array}$ & $\begin{array}{l}\text { CNN } \\
(2012 \text { ImageNet } \\
\text { pre-trained } \\
\text { VGG16) }\end{array}$ & $\begin{array}{l}251 \text { periapical } \\
\text { radiographs } \\
(500 \times 748 \text { pixel })\end{array}$ & $\begin{array}{l}\text { Classification } \\
\text { (dental caries, } \\
\text { periapical infection, } \\
\text { periodontitis) }\end{array}$ & Accuracy $=0.8846$ \\
\hline
\end{tabular}




\begin{tabular}{|c|c|c|c|c|}
\hline $\begin{array}{l}\text { Ekert } \\
2019^{44}\end{array}$ & CNN & $\begin{array}{l}1,331 \text { cropped } \\
\text { panoramic } \\
\text { radiographs of } 85 \\
\text { patients }(64 \times 64 \text { pixel })\end{array}$ & $\begin{array}{l}\text { Classification } \\
\text { (no attachment loss, } \\
\text { widened periodontal } \\
\text { ligament, clearly } \\
\text { detectable lesion) }\end{array}$ & $\begin{array}{l}\text { Recall }{ }^{\dagger}=0.74 \pm 0.19, \\
\text { Specificity }=0.94 \pm 0.04 \\
\text { Precision }{ }^{\dagger}=0.67 \pm 0.14, \\
N P V=0.95 \pm 0.04 \\
A U C=0.95 \pm 0.02 \\
\text { In all teeth, majority(6) } \\
\text { reference test condition }\end{array}$ \\
\hline $\begin{array}{l}\text { Yang } \\
2018^{82}\end{array}$ & $\begin{array}{l}\text { CNN(GoogLeNet } \\
\text { Inception v3) }\end{array}$ & $\begin{array}{l}196 \text { pairs of periapical } \\
\text { radiograph before and } \\
\text { after the treatments } \\
(96 \times 192 \text { pixel })\end{array}$ & $\begin{array}{l}\text { Classification } \\
\text { (getting better, } \\
\text { getting worse, have } \\
\text { no explicit change) }\end{array}$ & $\begin{array}{l}\text { Precision }=0.537, \\
\text { Recall }=0.490, \mathrm{~F} 1 \\
\text { score }=0.517\end{array}$ \\
\hline
\end{tabular}

\begin{tabular}{llll}
\hline 3.4. Detection of (pre)cancerous lesion & \\
\hline Uthoff & CNN & 170 pairs of & Classification \\
$2008^{39}$ & & $\begin{array}{l}\text { Autofluorescence } \\
\text { image and white light }\end{array}$ & $\begin{array}{l}\text { (cancerous and } \\
\text { pre-cancerous lesion, } \\
\end{array}$ \\
& image & not suspicious)
\end{tabular}

Remote specialist

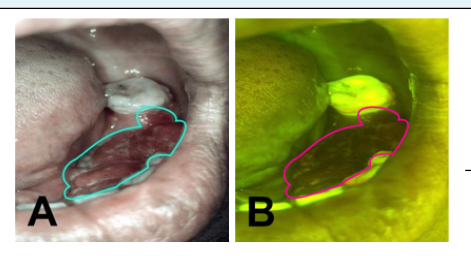

Precision $^{\dagger}=0.8767$, Recall $^{\dagger}=0.8500$, Specificity $=0.8875$, $\mathrm{NPV}=0.8549, \mathrm{AUC}=0.908$;

Precision $^{\dagger}=0.9494$, Recall ${ }^{\dagger}=0.9259$, Specificity $=0.8667$, $\mathrm{NPV}=0.8125$

\begin{tabular}{lll}
\hline $\begin{array}{l}\text { Aubreville } \\
2017^{40}\end{array}$ & $\begin{array}{l}\text { CNN } \\
+ \text { Probability fusion }\end{array}$ & $\begin{array}{l}\text { 165,774 patches } \\
\text { extracted from } 7,894 \\
\\
\end{array}$ \\
& grayscale confocal \\
& laser endomicroscopy \\
& video frames of the \\
& inner lower labium, \\
& the upper alveolar \\
& ridge, the hard palate \\
& $(80 \times 80$ pixel $)$
\end{tabular}
grayscale confocal laser endomicroscopy ideo frames of the $(80 \times 80$ pixel $)$

\begin{tabular}{|c|c|c|}
\hline \multirow[t]{4}{*}{$\begin{array}{l}\text { Forslid } \\
2017^{41}\end{array}$} & \multirow[t]{4}{*}{$\begin{array}{l}\text { CNN } \\
\text { (VGG16, ResNet18) }\end{array}$} & $\begin{array}{l}\text { Oral dataset } 1 \\
(15 \text { microscopic cell } \\
\text { images taken at } \times 20 \\
\text { magnification })\end{array}$ \\
\hline & & $\begin{array}{l}\text { Oral dataset } 2 \\
(15 \text { microscopic cell } \\
\text { images taken at } \times 20 \\
\text { magnification })\end{array}$ \\
\hline & & $\begin{array}{l}\text { CerviSCAN dataset } \\
(12,043 \text { microscopic } \\
\text { cell images taken at } \\
\times 40 \text { magnification) }\end{array}$ \\
\hline & & $\begin{array}{l}\text { Herlev dataset } \\
\text { (917 microscopic cell } \\
\text { images) }\end{array}$ \\
\hline
\end{tabular}

\section{Classification} (normal, cancerous)

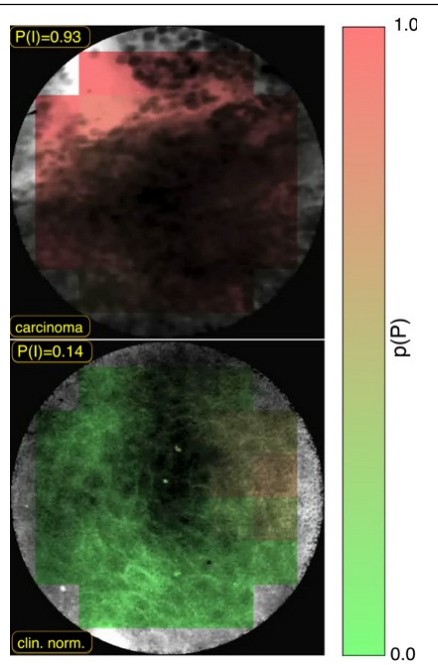

\begin{tabular}{|c|c|c|}
\hline \multirow{17}{*}{$\begin{array}{l}\text { Classification } \\
\text { (healthy, tumor) }\end{array}$} & VGG16 & ResNet18 \\
\hline & \multirow{4}{*}{$\begin{array}{l}\text { Accuracy }=80.66 \pm 3.00, \\
\text { Precision }=75.04 \pm 7.68, \\
\text { Recall }=80.68 \pm 3.05, \\
\text { F1 score }=77.68 \pm 5.28\end{array}$} & \multirow{4}{*}{$\begin{array}{l}\text { Accuracy }=78.34 \pm 2.37 \\
\text { Precision }=72.48 \pm 4.46, \\
\text { Recall }=79.00 \pm 3.37, \\
\text { F1 score } 75.51 \pm 3.17\end{array}$} \\
\hline & & \\
\hline & & \\
\hline & & \\
\hline & Accuracy $=80.83 \pm 2.55$, & Accuracy $=82.39 \pm 2.05$ \\
\hline & Precision $=82.41 \pm 2.55$ & Precision $=82.45 \pm 2.38$ \\
\hline & Recall $=79.79 \pm 3.75$ & Rcall $=82.58 \pm 1.92$ \\
\hline & F1 score $=81.07 \pm 3.17$ & F1 score $82.51 \pm 2.15$ \\
\hline & Accuracy $=84.20 \pm 0.86$, & Accuracy $=84.45 \pm 0.46$ \\
\hline & Precision $=84.35 \pm 0.97$ & Precision $=84.64 \pm 0.38$ \\
\hline & Recall $=84.20 \pm 0.86$ & Rcall $=84.45 \pm 0.47$ \\
\hline & F1 score $=84.28 \pm 0.91$ & F1 score $84.28 \pm 0.91$ \\
\hline & Accuracy $=86.56 \pm 3.18$ & Accuracy $=86.45 \pm 3.81$, \\
\hline & Precision $=85.94 \pm 6.98$, & Precision $=82.45 \pm 5.11$, \\
\hline & Recall $=79.04 \pm 3.81$ & Recall $=84.45 \pm 2.16$ \\
\hline & F1 score $=82.16 \pm 3.85$ & F1 score $83.36 \pm 3.65$ \\
\hline
\end{tabular}




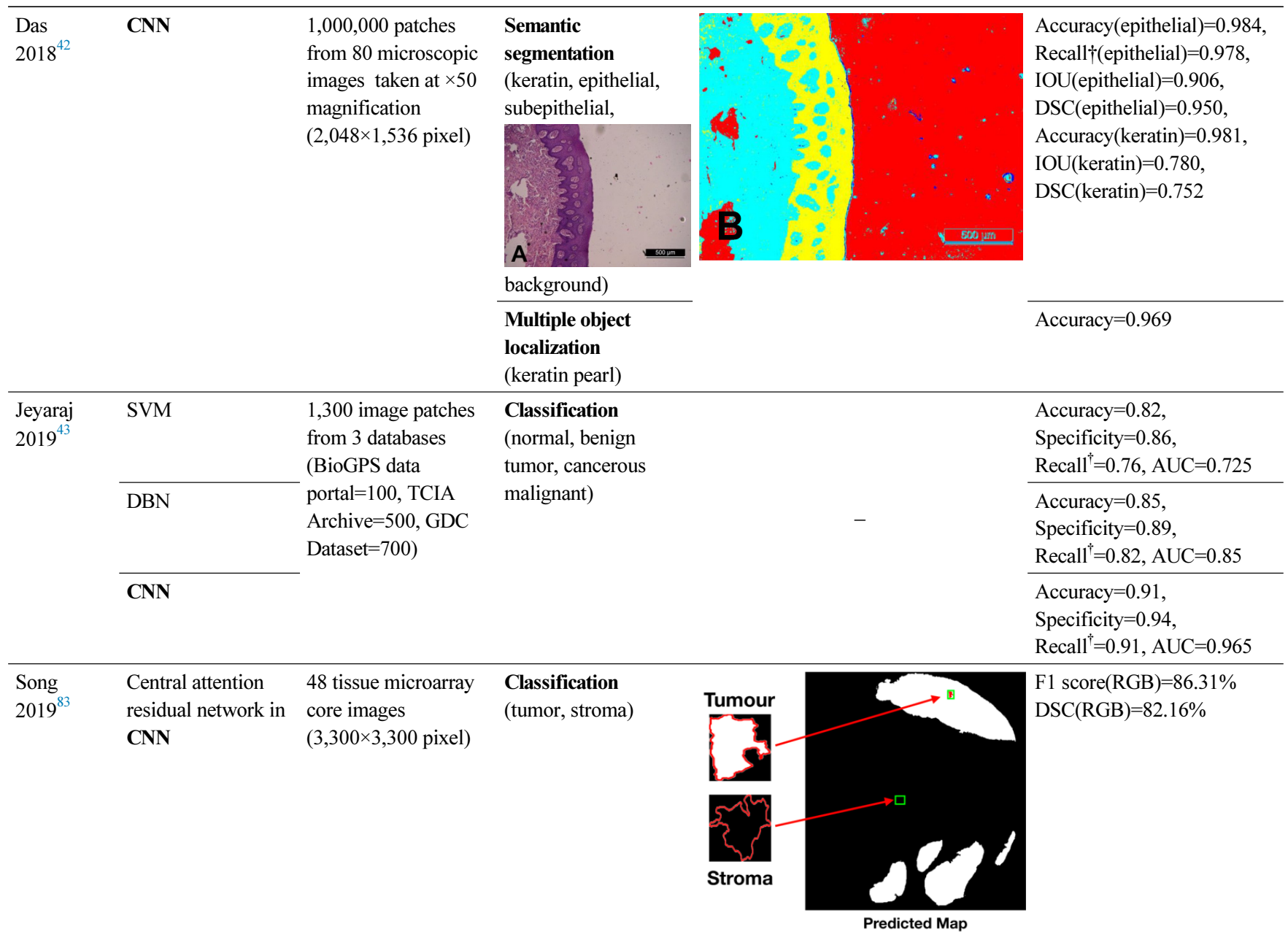

3.5. Detection of other disease

\begin{tabular}{|c|c|c|c|c|}
\hline \multirow[t]{3}{*}{$\begin{array}{l}\text { Murata } \\
2019^{45}\end{array}$} & CNN(AlexNet) & $\begin{array}{l}800 \text { cropped } \\
\text { panoramic } \\
\text { radiographs of } \\
\text { maxillary sinus } \\
(200 \times 200 \text { pixel })\end{array}$ & $\begin{array}{l}\text { Classification } \\
\text { (healthy, inflamed) }\end{array}$ & $\begin{array}{l}\text { Accuracy }=0.875, \\
\text { Recall }{ }^{\dagger}=0.867, \\
\text { Specificity }=0.883, \\
\text { Precision }{ }^{\dagger}=0.881, \\
\text { NPV }=0.869, \text { AUC }=0.875\end{array}$ \\
\hline & Radiologist & & & $\begin{array}{l}\text { Accuracy }=0.896, \\
\text { Recall }{ }^{\dagger}=0.900, \\
\text { Specificity }=0.892, \\
\text { Precision }{ }^{\dagger}=0.893, \\
\text { NPV }=0.899, \text { AUC }=0.896\end{array}$ \\
\hline & Dental residents & & & $\begin{array}{l}\text { Accuracy }=0.767, \\
\text { Recall }=0.783, \\
\text { Specificity }=0.750, \\
\text { Precision }{ }^{\dagger}=0.758, \\
\text { NPV }=0.776, \text { AUC }=0.767\end{array}$ \\
\hline $\begin{array}{l}\text { De Dumast } \\
2018^{46}\end{array}$ & $\mathrm{NN}$ & $\begin{array}{l}293 \text { condyle images } \\
\text { from reconstructed } \\
\text { CBCT }\end{array}$ & $\begin{array}{l}\text { Classification(close } \\
\text { to normal[control], } \\
\text { close to normal } \\
\text { [osteoarthritis], } \\
\text { degeneration } \\
1,2,3,4-5)\end{array}$ & $\begin{array}{l}\text { In confusion matrix, } \\
\text { Accuracy }=0.441, \\
\text { Accuracy(including adjacent } \\
1 \text { cell around true positive } \\
\text { cell })=0.912\end{array}$ \\
\hline
\end{tabular}




\begin{tabular}{|c|c|c|c|c|}
\hline \multirow[t]{3}{*}{$\begin{array}{l}\text { Kise } \\
2019^{47}\end{array}$} & CNN(AlexNet) & \multirow[t]{3}{*}{$\begin{array}{l}500 \text { cropped CT } \\
\text { images of parotid } \\
\text { gland in } 25 \text { patients }\end{array}$} & \multirow[t]{3}{*}{$\begin{array}{l}\text { Classification } \\
\text { (normal, Sjögren } \\
\text { syndrome) }\end{array}$} & $\begin{array}{l}\text { Accuracy }=0.96, \text { Recall }^{\dagger}=1, \\
\text { Specificity }=0.92, \\
\text { AUC }=0.960\end{array}$ \\
\hline & $\begin{array}{l}\text { Experienced } \\
\text { radiologist }\end{array}$ & & & $\begin{array}{l}\text { Accuracy }=0.983, \\
\text { Recall }{ }^{\dagger}=0.993, \\
\text { Specificity }=0.779, \\
\text { AUC }=0.996\end{array}$ \\
\hline & $\begin{array}{l}\text { Inexperienced } \\
\text { radiologist }\end{array}$ & & & $\begin{array}{l}\text { Accuracy }=0.835, \\
\text { Recall }{ }^{\dagger}=0.779, \\
\text { Specificity }=0.892, \\
\text { AUC }=0.997\end{array}$ \\
\hline $\begin{array}{l}\text { Chu } \\
2018^{48}\end{array}$ & $\begin{array}{l}\text { Octuplet Siamese } \\
\text { network with } \\
\text { 2-stage fine tuning }\end{array}$ & $\begin{array}{l}864 \text { cropped } \\
\text { panoramic } \\
\text { radiographs } \\
(50 \times 50 \text { pixel })\end{array}$ & $\begin{array}{l}\text { Classification } \\
\text { (normal, osteoporosis) }\end{array}$ & Accuracy $=0.898$ \\
\hline $\begin{array}{l}\text { Kats } \\
2019^{49}\end{array}$ & $\begin{array}{l}\text { Faster R-CNN } \\
\text { (ResNet101) }\end{array}$ & $\begin{array}{l}65 \text { panoramic } \\
\text { radiographs }\end{array}$ & $\begin{array}{l}\text { Multiple object } \\
\text { localization } \\
\text { (atherosclerotic } \\
\text { carotid plaques) }\end{array}$ & $\begin{array}{l}\text { Accuracy }=0.83, \\
\text { Recall }^{\dagger}=0.75 \\
\text { Specificity }=0.80, \text { AUC }=0.83\end{array}$ \\
\hline
\end{tabular}

\section{Evaluation of facial esthetics, detection of cephalometric landmarks}

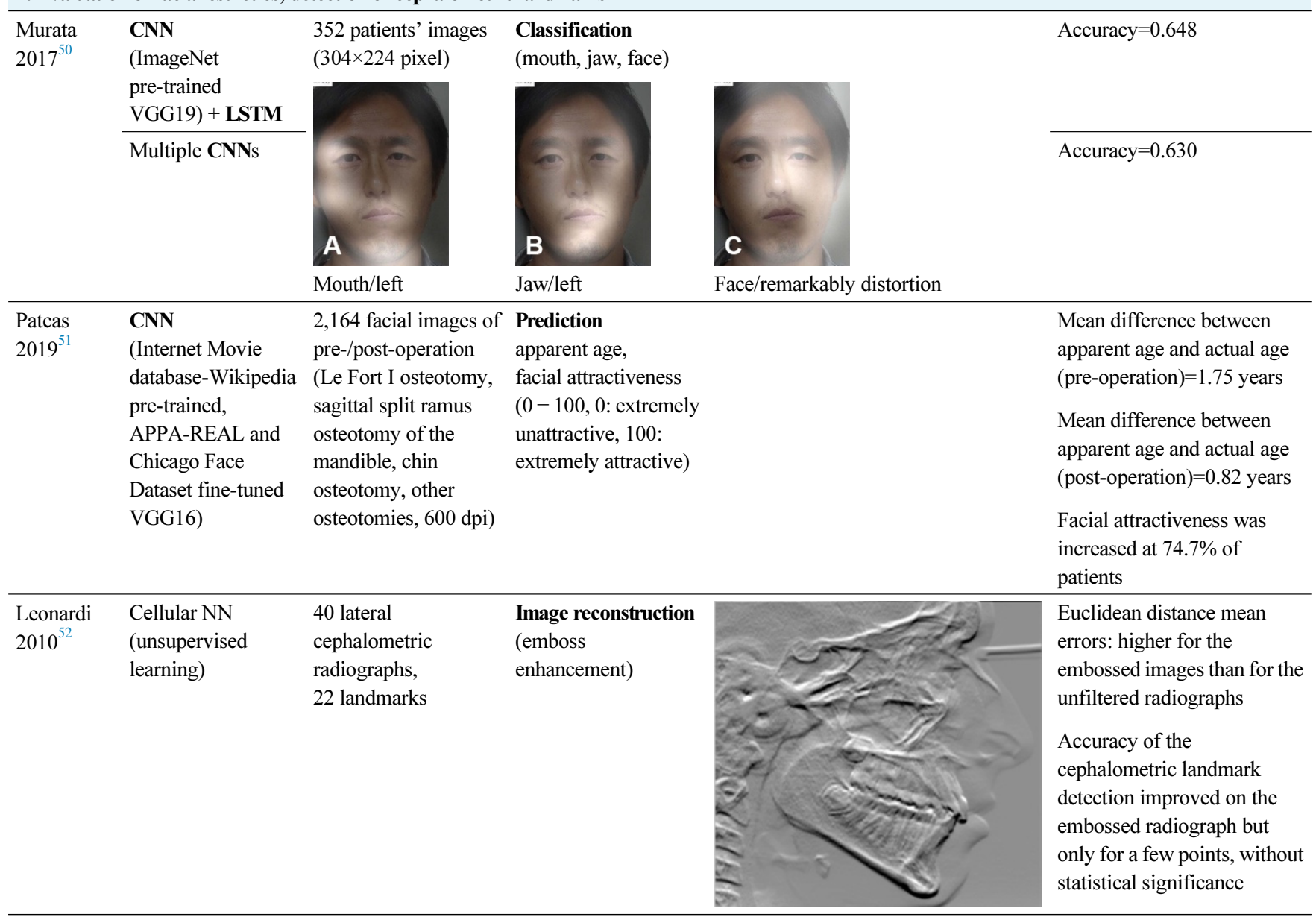




\begin{tabular}{|c|c|c|c|c|}
\hline $\begin{array}{l}\text { Qian } \\
2019^{53}\end{array}$ & $\begin{array}{l}\text { Faster R-CNN with } \\
\text { VOC } 2012 \\
\text { pre-trained } \\
\text { ResNet50) }\end{array}$ & $\begin{array}{l}400 \text { lateral } \\
\text { cephalometric } \\
\text { radiographs }\end{array}$ & $\begin{array}{l}\text { Multiple object } \\
\text { localization } \\
\text { (19 landmarks) }\end{array}$ & $\begin{array}{l}\text { Accuracy(test } 1)=0.825 \text {, } \\
\text { Accuracy(test } 2)=0.724 \\
\text { Landmark can be classified } \\
\text { as a 'accurate' on only if the } \\
\text { distance between a detected } \\
\text { landmark and its ground truth } \\
\text { is less than } 2 \mathrm{~mm} \text {. }\end{array}$ \\
\hline \multirow[t]{2}{*}{$\begin{array}{l}\text { Torosdagli } \\
2019^{54}\end{array}$} & \multirow[t]{2}{*}{$\begin{array}{l}\text { CNN } \\
\text { (U-Net, deep } \\
\text { geodesic learning), } \\
\text { LSTM }\end{array}$} & \multirow[t]{2}{*}{$\begin{array}{l}25,600 \text { slices from } 50 \\
3 \mathrm{D} \text { reconstruced } \\
\text { CBCT }\end{array}$} & $\begin{array}{l}\text { Segmentation } \\
\text { (mandible) }\end{array}$ & $\begin{array}{l}\mathrm{DSC}=0.9386, \text { Hausdorff } \\
\text { distance }=5.47\end{array}$ \\
\hline & & & $\begin{array}{l}\text { Multiple object } \\
\text { localization } \\
\text { (landmarks on } \\
\text { mandible) }\end{array}$ & $\begin{array}{l}\text { Mean error }(\mathrm{mm}) \text { : } \\
\text { coronoid process }(\text { left })=0, \\
\text { coronoid } \\
\text { process }(\text { right })=0.45, \\
\text { condyle }(\text { left })=0.33, \\
\text { condyle }(\text { right })=0.07, \\
\text { menton }=0.03 \text {, gnathion }=0.49 \text {, } \\
\text { pogonion }=1.54, \text { B-point }=0.33 \text {, } \\
\text { infradentale }=0.52\end{array}$ \\
\hline \multicolumn{5}{|c|}{ 5. Fabrication of prosthesis } \\
\hline \multirow[t]{2}{*}{$\begin{array}{l}\text { Shen } \\
2019^{84}\end{array}$} & \multirow[t]{2}{*}{ CNN(U-Net) } & \multirow[t]{2}{*}{$\begin{array}{l}77 \text { single crown } \\
\text { models }\end{array}$} & $\begin{array}{l}\text { Prediction } \\
\text { (deformation, } \\
\text { translation, scaling } \\
\text { down, rotation) } \\
\end{array}$ & $\begin{array}{l}F 1 \text { score }(\text { deformation })=0.9614, \\
F 1 \text { score }(\text { scaling down })=0.9408, \\
F 1 \text { score }(\text { translation })=0.9386, \\
F 1 \text { score }(\text { rotation })=0.9387\end{array}$ \\
\hline & & & $\begin{array}{l}\text { Image reconstruction } \\
\text { (compensating } \\
\text { deformation, } \\
\text { translation, scaling } \\
\text { down, rotation) }\end{array}$ & $\begin{array}{l}\text { F1 score }(\text { deformation })=0.9699, \\
\text { F1 score }(\text { scaling down })=0.9488, \\
\text { F1 score }(\text { translation })=0.9517, \\
\text { F1 score }(\text { rotation })=0.9417\end{array}$ \\
\hline \multirow[t]{2}{*}{$\begin{array}{l}\text { Shen } \\
2019^{55}\end{array}$} & \multirow[t]{2}{*}{ CNN(U-Net) } & \multirow[t]{2}{*}{$\begin{array}{l}28,433 \text { slices from } 71 \\
3 \mathrm{D} \text { single crown } \\
\text { models } \\
(256 \times 256 \text { pixel })\end{array}$} & $\begin{array}{l}\text { Prediction } \\
\text { (deformation, scaling } \\
\text { down, rotation) }\end{array}$ & $\begin{array}{l}\text { Recall }(\text { deformation})=1.000, \\
\text { Recall(scaling down) }=0.984, \\
\text { Recall(rotation) }=0.982, \\
\text { Precision }(\text { deformation })=1.00 \\
0, \text { Precision }(\text { scaling } \\
\text { down })=0.993, \\
\text { Precision }(\text { rotation })=0.978, \\
\text { F1 score }(\text { deformation })=1.000, \\
\text { F1 score }(\text { scaling down })=0.989, \\
\text { F1 score }(\text { rotation })=0.980\end{array}$ \\
\hline & & & $\begin{array}{l}\text { Image reconstruction } \\
\text { (compensating } \\
\text { deformation, scaling } \\
\text { down, rotation) }\end{array}$ & $\begin{array}{l}\text { Recall }(\text { deformation })=1.000, \\
\text { Recall }(\text { scaling down })=0.993, \\
\text { Recall }(\text { rotation })=0.983, \\
\text { Precision }(\text { deformation })=1.000, \\
\text { Precision }(\text { scalin down })=0.991, \\
\text { Precision }(\text { rotation })=0.980, \\
F 1 \text { score }(\text { deformation })=1.000, \\
F 1 \text { score }(\text { scaling down })=0.992, \\
F 1 \text { score }(\text { rotation })=0.982\end{array}$ \\
\hline
\end{tabular}




\begin{tabular}{|c|c|c|c|c|}
\hline $\begin{array}{l}\text { Yama } \\
\text {-guchi } \\
2019^{56}\end{array}$ & CNN & $\begin{array}{l}8,640 \text { 3D scan images } \\
\text { of study model } \\
(100 \times 100 \text { pixel })\end{array}$ & $\begin{array}{l}\text { Classification } \\
\text { (trouble-free, } \\
\text { debonding) }\end{array}$ & $\begin{array}{l}\text { Accuracy }=0.985, \\
\text { Precision }=0.970, \text { Recall }=1, \\
\text { F1 score }=0.985, \text { AUC }=0.098\end{array}$ \\
\hline \multirow[t]{3}{*}{$\begin{array}{l}\text { Zhang } \\
2019^{57}\end{array}$} & \multirow[t]{3}{*}{$\begin{array}{l}\text { CNN(sparse octree } \\
\text { structure, } \\
\text { voxel-based) }\end{array}$} & $\begin{array}{l}\text { From } 380 \text { preparation } \\
\text { models: } \\
\text { dataset A: no rotation }\end{array}$ & \multirow[t]{3}{*}{ Segmentation(preparation line) } & $\begin{array}{l}\text { Accuracy }=0.9062, \\
\text { Recall }{ }^{\dagger}=0.9318, \\
\text { Specificity }=0.9458\end{array}$ \\
\hline & & $\begin{array}{l}\text { dataset B: } 60^{\circ} \times 5 \\
\text { rotations }\end{array}$ & & $\begin{array}{l}\text { Accuracy }=0.9558, \\
\text { Recall }^{\dagger}=0.9590, \\
\text { Specificity }=0.9521\end{array}$ \\
\hline & & $\begin{array}{l}\text { dataset } \mathrm{C}: 30^{\circ} \times 12 \\
\text { rotations }\end{array}$ & & $\begin{array}{l}\text { Accuracy }=0.9743, \\
\text { Recall }^{\dagger}=0.9759, \\
\text { Specificity }=0.9732\end{array}$ \\
\hline \multirow[t]{2}{*}{$\begin{array}{l}\text { Zhao } \\
2019^{58}\end{array}$} & \multirow[t]{2}{*}{$\begin{array}{l}\text { Convolutional } \\
\text { auto-encoder }\end{array}$} & \multirow{2}{*}{$\begin{array}{l}39,424 \text { models } \\
\text { augmented by rotating } \\
77 \text { dental crown } \\
\text { models }\end{array}$} & $\begin{array}{l}\text { Prediction(nonlinear } \\
\text { deformation) }\end{array}$ & $\begin{array}{l}\text { F1 score(nonlinear } \\
\text { deformation, resolution } \\
64 \times 64 \times 64)=0.9684\end{array}$ \\
\hline & & & $\begin{array}{l}\text { Image reconstruction } \\
\text { (compensation) }\end{array}$ & $\begin{array}{l}\text { F1 score (nonlinear } \\
\text { deformation, resolution } \\
64 \times 64 \times 64)=0.9755 \\
\text { F1 score before } \\
\text { compensation }(0.7782) \text { was } \\
\text { increased } \\
\text { after compensation }(0.9530)\end{array}$ \\
\hline \multicolumn{5}{|l|}{ 6. Others } \\
\hline $\begin{array}{l}\text { Milošević } \\
2019^{59}\end{array}$ & $\begin{array}{l}\text { CNN(ImageNet } \\
\text { pre-trained } \\
\text { VGG16) }\end{array}$ & $\begin{array}{l}4,000 \text { panoramic } \\
\text { radiographs }(\text { female }= \\
58.8 \%, \text { male }=41.2 \%)\end{array}$ & $\begin{array}{l}\text { Classification } \\
\text { (female, male) }\end{array}$ & $\begin{array}{l}\text { Accuracy }=96.87 \pm 0.96 \% \\
\text { (filter }=256, \text { unit }=128, \\
\text { without attention } \\
\text { mechanism) }\end{array}$ \\
\hline $\begin{array}{l}\text { Ilić } \\
2019^{60}\end{array}$ & $\begin{array}{l}\text { CNN(Pre-trained } \\
\text { VGG16) }\end{array}$ & $\begin{array}{l}4,155 \text { panoramic } \\
\text { radiographs } \\
(512 \times 512 \text { pixel })\end{array}$ & $\begin{array}{l}\text { Classification } \\
\text { (female, male) }\end{array}$ & $\begin{array}{l}\text { Accuracy }=94.3 \% \text { (over } 80 \\
\text { years }=50 \%) \text {, } \\
\text { Testing time }=0.018 \text { seconds }\end{array}$ \\
\hline \multirow[t]{4}{*}{$\begin{array}{l}\text { Alarifi } \\
2018^{85}\end{array}$} & Radial basis NN & \multirow{4}{*}{$\begin{array}{l}\text { Patient self-behavior, } \\
\text { health conditions, } \\
\text { attitude information }\end{array}$} & \multirow[t]{4}{*}{$\begin{array}{l}\text { Prediction } \\
\text { (implant success) }\end{array}$} & $\begin{array}{l}\text { Recall }^{\dagger}=0.8478 \\
\text { Specificity }=0.8678\end{array}$ \\
\hline & $\begin{array}{l}\text { General regression } \\
\text { NN }\end{array}$ & & & $\begin{array}{l}\text { Recall }^{\dagger}=0.9216 \\
\text { Specificity }=0.9351\end{array}$ \\
\hline & Associative NN & & & $\begin{array}{l}\text { Recall }^{\dagger}=0.9417 \\
\text { Specificity }=0.9482\end{array}$ \\
\hline & $\begin{array}{l}\text { Memetic search } \\
\text { optimization along } \\
\text { with genetic scale } \\
\text { RNN }\end{array}$ & & & $\begin{array}{l}\text { Recall }^{\dagger}=0.9763 \\
\text { Specificity }=0.9828\end{array}$ \\
\hline
\end{tabular}




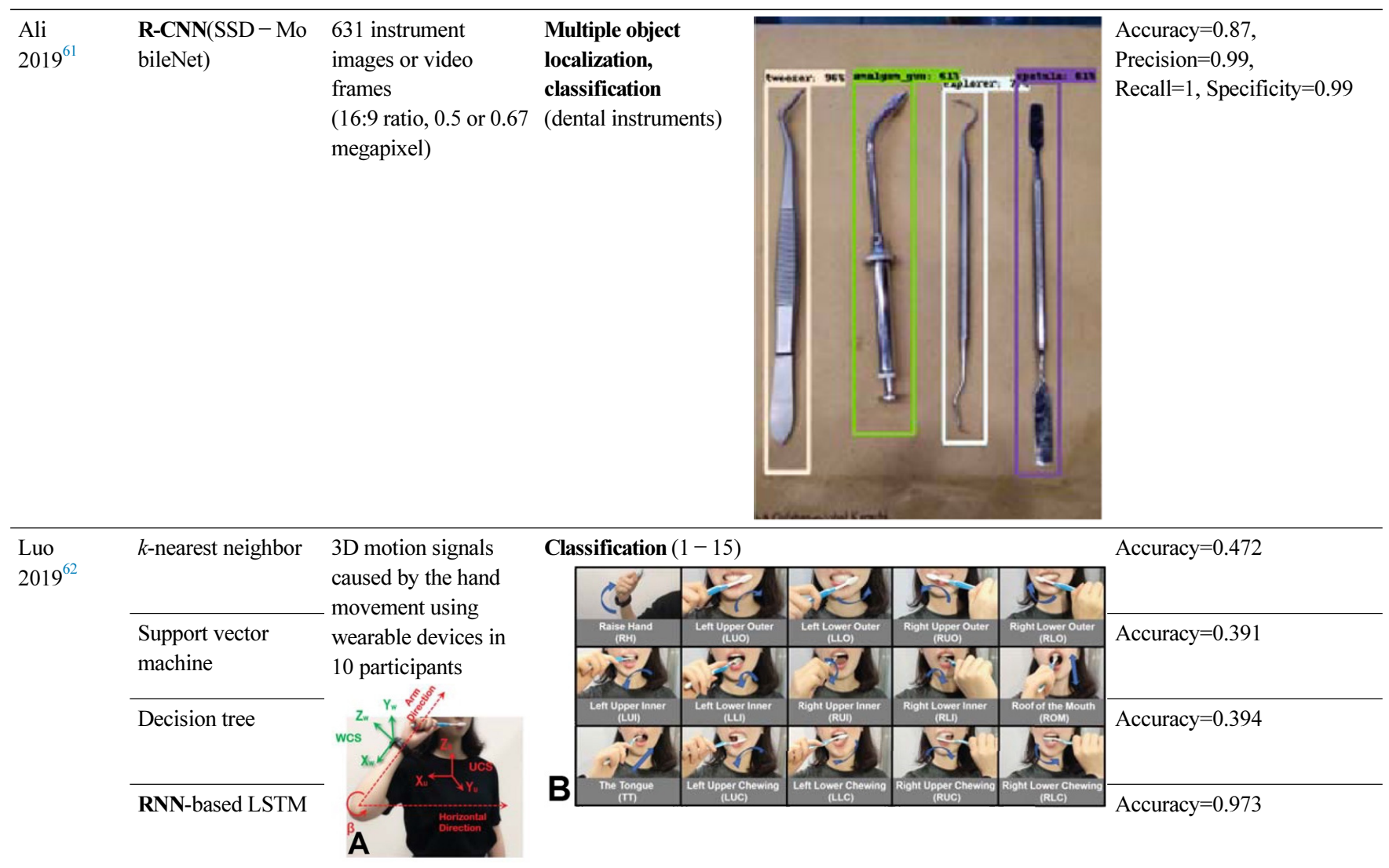

3D: three-dimensional; AUC: area under curve; CAD/CAM: computer-aided design/computer-aided manufacturing; CBCT: cone-beam computed tomography; CNN: convolutional neural network; CT: computed tomography; DSC: dice similarity coefficient; GAN: generative adversarial network; HU: Hounsfield unit; ICDAS: international caries detection and assessment system; IOU: intersection-over-union; LSTM: long short-term memory models; LRTV: low-rank and total variation regularizations; m-WGAN: modified-Wasserstein generative adversarial network; NN: neural network; NPV: negative predictive value; PSNR: peak signal-to-noise ratio; R-CNN: region-based convolutional neural network; SSI: structure similarity index; SRR: super-resolution method; TF-SISR: tensor factorization with 3D single image super-resolution.

*: refers to the data unanimously classified by 6 specialists

${ }^{\dagger}$ : sensitivity and positive predictive value were replaced by recall and precision, respectively.

1) Detection of teeth and adjacent anatomical structures

The selected studies in this category can be subdivided into three groups: tooth detection, tooth numbering, tooth segmentation, and bone segmentation. For tooth detection and tooth numbering, panoramic radiographs and cone-beam computed tomography (CBCT) images were used. For multiple object localization of teeth, the precision was reported to be in the range $0.900^{12}-0.995^{13}$ and the recall in the range $0.983^{14}-0.994^{13}$. The precision of tooth numbering was reported to be in the range $0.715^{12}-$ $0.958^{14}$ and the recall in the range $0.782^{12}-0.980^{13}$.

Tooth segmentation has been attempted for teeth in panoramic radiographs ${ }^{15}$, third molars ${ }^{16}$, and dental mode ${ }^{17,18}$. Algorithms for segmenting bone appearances from $\mathrm{CBCT}^{19}$ and oral ultrasound images ${ }^{20}$ have been also proposed. Two studies that classified the developmental stages of third molars reported accuracies of $0.51^{21}$ and $0.61^{16}$. 
2) Image quality enhancement

For image quality enhancement, studies on the reduction of blur, noise, and metal artifacts as well as on the super-resolution have been conducted. Du et al. corrected blurs in the center of images using an algorithm trained using 5,166 panoramic radiographs taken at positions $\pm 20 \mathrm{~mm}$ from the ideal position and the misalignment length $(\mathrm{mm})$, and they reported a maximum absolute error below $1.5 \mathrm{~mm} .{ }^{22}$ Liang et al. reconstructed computed tomography (CT) images using three algorithms and reported improved root mean squared error and structure similarity index compared with the values measured in original CT images. ${ }^{23} \mathrm{Hu}$ compared a GAN, CNN, and modified GAN using Wasserstein distance and reported that the latter was most effective in the noise reduction from CBCT images. ${ }^{24}$ Hegazy reported improved the relative error by $5.7 \%$, and the standardized absolute difference by $8.2 \%$ using modified U-net algorithm compared to the conventional method.$^{25}$ Dinkla et al. introduced a U-net-based algorithm that synthesizes CT images with no metal artifacts from T2-weighted magnetic resonance imaging (MRI). ${ }^{26}$ Hatvani et al. reported a dice similarity index of 0.90 and a mean difference of $9.87 \%$ comparing crosssectional area of root canal system of CBCT applied tensor factorization super-resolution algorithm with that of micro $\mathrm{CT}^{27}{ }^{27}$ They also attempted super-resolution using a subpixel network, reporting a dice similarity index of 0.91 and a mean difference root volume of $6.07 \%$ compared with micro $\mathrm{CT}^{28}$

\section{3) Disease detection}

Target diseases include tooth caries, ${ }^{29-34}$ periodontal disease, ${ }^{34-38}$ precancerous lesions, ${ }^{39-43}$ periapical diseases, ${ }^{33,44}$ dental fluorosis, ${ }^{34}$ maxillary sinusitis, ${ }^{45}$ osteoarthritis,${ }^{46}$ Sjögren's syndrome,${ }^{47}$ and osteoporosis. ${ }^{48}$ An algorithm for detecting atherosclerotic carotid plaque in panoramic radiographs has been suggested. ${ }^{49}$

4) Evaluation of facial esthetics and localization of cephalometric landmarks

Algorithms for evaluating various images such as facial photographs, lateral cephalometric radiographs, and CBCT images have been proposed. Murata et al. developed an algorithm for classifying the asymmetry and/or discrepancy of crow's feet, nose, lips, and chin from input frontal face image, and reported an mean accuracy of $64.8 \%{ }^{50}$ Patcas et al. trained CNN-based algorithm to estimate apparent age and facial attractiveness score ( $0-100$ points) using various facial image datasets, and they reported the increased facial attractiveness score (mean difference $=1.22,95 \%$ confidence interval: $0.81,1.63$ ) and decreased apparent age (mean difference $=-0.93,95 \%$ confidence interval: $-1.50,-0.36$ ) after orthognathic surgery. ${ }^{51}$ Leonardi synthesized embossed images from lateral cephalometric radiograph for enhanced visibility but reported insignificant improvement of reading accuracy. ${ }^{52}$ Qian et al.proposed 
faster R-CNN method for cephalometric landmark detection. After trained with 150 photographs of 19 types of cephalometric landmarks that were manually localized by expert orthodontist, the method localized landmarks within $2 \mathrm{~mm}$ of the landmark located by the orthodontist at $72.4-82.5 \%{ }^{53}$ The cephalometric landmarks on the mandible (menton, gnathion, pogonion, B-point, infradentale, coronoid process, condyle head) were localized after segmenting the mandible on the $3 \mathrm{D}$ reconstructed $\mathrm{CBCT}$, and an mean error was reported to less than $1 \mathrm{~mm}$ except for pogonion. ${ }^{54}$

\section{5) Fabrication of prosthesis}

Shen et al. proposed algorithm for predicting and compensating for errors in the cross section of single crown additively manufactured using the 3D printer, and they reported improved F1 scores (translation: $0.6894 \rightarrow 0.9995$, scaling down: $0.7188 \rightarrow 0.9893$, rotation: $0.8906 \rightarrow 0.9671) .{ }^{55}$ Yamaguchi developed an algorithm that evaluates the scan data of an abutment preparation model and classified models with a high possibility of debonding and a low possibility (trouble-free), and they reported an accuracy of $98.5 \%{ }^{56}$ In addition, an algorithm that predicts the crown margin in a 3D-scanned abutment preparation model ${ }^{57}$ and an algorithm for predicting the nonlinear deformation of $3 \mathrm{D}$ printed crowns from the scan data of an abutment preparation model have been suggested. ${ }^{58}$

\section{6) Others}

Milosevic and Ilic designed an algorithm for determining sex from panoramic radiographs and reported accuracies of $96.87 \pm 0.96 \%{ }^{59}$ and $94.3 \%,{ }^{60}$ respectively. Ali et al. reported on an algorithm for localizing and classifying dental instruments in image ${ }^{61}$ Luo et al. collected 3D motion signals in daily life using wearable devices and tested four algorithms classifying tooth brushing time and 15 tooth brushing motions. In terms of classifying tooth brushing motions, they reported an mean classification accuracy of $97.3 \%$ using the RNN-based algorithm. ${ }^{62}$

\section{IV . Discussion}

\section{Principle of Machine Learning}

The basic approach of machine learning is to set a loss function for the difference between the predicted value $(\hat{y})$ and the groud truth $(y)$ and determine the global minimum of the loss function based on the fact that accuracy improves as the loss function decreases (Fig. 11). ${ }^{63}$ One representative example is the least squares method, which squares the differences between each predicted value and the ground truth 
and minimizes the sum of these differences. The loss function is the mean of the squared differences between the predicted values and the ground truth.

$$
\text { loss function }=\frac{1}{m} \sum_{i=1}^{m}\left(y_{i}-\hat{y}_{i}\right)^{2}
$$

A basic algorithm for determining the global minimum of the loss function is gradient descent. A smaller input value $(\chi)$ is substituted if the gradient of the loss function is positive, and a larger input value is substituted if the gradient is negative, thus converging to the minimum.

\section{Development of Deep Learning}

Deep learning is a field of machine learning (Fig. 12) and refers to deep artificial neural networks with two or more hidden layers besides the output layer. An artificial neural network is an interconnected

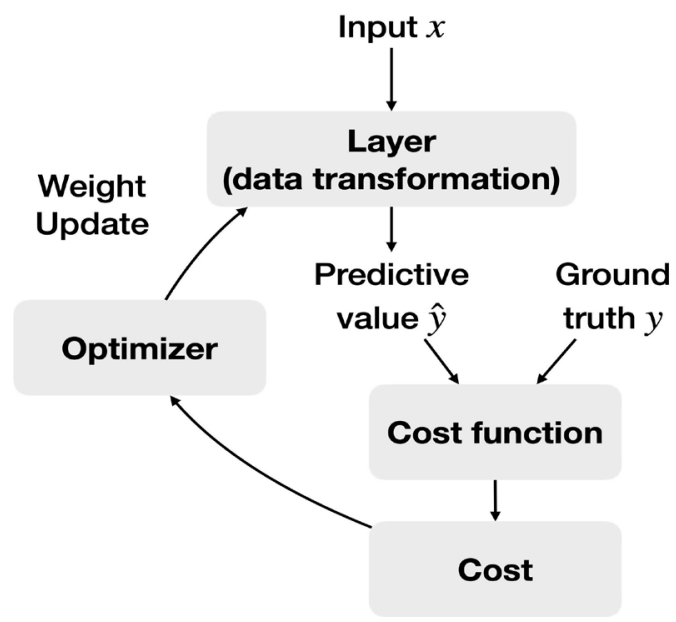

Fig. 11. Schematic diagram showing the training process of machine learning. Adapted from "Deep Learning mit Python und Keras: Das Praxis-Handbuch vom Entwickler der Keras-Bibliothek" by Chollet F., Copyright 2018 by MITP-Verlags GmbH \& Co. KG.

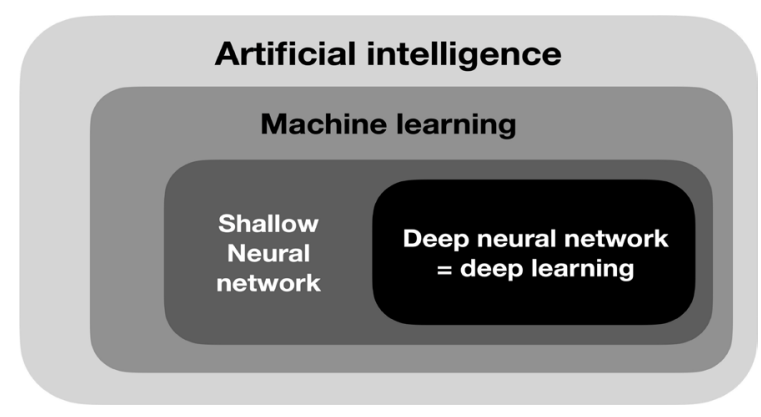

Fig. 12. Diagram showing the relationship between artificial intelligence, machine learning, and deep learning. Adapted from "Deep Learning mit Python und Keras: Das Praxis-Handbuch vom Entwickler der Keras-Bibliothek" by Chollet F., Copyrights 2018 by MITP-Verlags GmbH \& Co. KG. 
group of artificial neurons that perform computations by imitating the brain structure. It started with a simple neural network model with propositional logic, ${ }^{64}$ and artificial neurons called perceptrons ${ }^{65}$ were introduced. However, the exclusive OR operation could not be performed with a single-layer perceptron, ${ }^{66}$ and this problem was solved by developing the backpropagation algorithm for training the multi-layer perceptron. ${ }^{67,68}$ Current deep learning has been improved in performance through the development of a new activation function ${ }^{69}$ to solve the problem of vanishing gradient, while passing through the deep layer of the neural network, optimization of the weighted initialization, ${ }^{70-72}$ and dropout ${ }^{2}$ for preventing overfitting.

\section{Characteristics and Applicability of the Selected Studies}

For application of deep learning to dentistry, an algorithm for detecting multiple objects is required due to the nature of the dental anatomy that multiple teeth are distributed in a single image. For object localization, the sliding window technique was used in early research, which moves the windows of various ratios and sizes in the image in small increments. Later, the object localization became faster with the introduction of the region proposal network embedded in the neural network. Studies on teeth classification performance show slightly different results. To maintain high accuracy even in complex situations such as prosthesis, tooth defect, or mixed dentition, data in various situations need to be trained sufficiently.

If automatic tooth numbering algorithm is combined with classification algorithms for tooth caries, periodontal disease, and root apex disease, it can instantly provide useful clinical information by detecting abnormalities of each tooth. This can be highly beneficial in forensic dentistry as well. Although sex determination using panoramic radiographs shows lower accuracy $\left(94.3 \%{ }^{60}, 96.87 \%{ }^{59}\right)$ than using the total skeleton $(100 \%)$; however, it has the additional advantages that people with partial bones can be analyzed and their dental records can be compared. Analyzing the development stage of third molars can be one of age estimation methods with analyzing hand-wrist radiograph or cervical vertebral maturation in panoramic radiograph.

Studies related to image quality enhancement of dental images mainly have been conducted for $\mathrm{CBCT}$, and development in three directions is anticipated. First, removal of noise due to the scattering of low-energy X-rays, which is the problem of low-dose CT, could increase the number of allowable shots while lowering the radiation exposure of patients, while also maintaining the image quality similar to that of normal dose CT. Second, reducing metal artifacts in the images could be considerably helpful for reading the CT images of patients who have several implants or metal fixed prostheses. For extreme 
example, Dinkla et al. proposed CNN-based method that synthesizes CT images similar to the existing CT from T2-weighted MRI with no radiation exposure and metal artifacts. Third, high-resolution images can be obtained by applying a super-resolution algorithm to conventional CBCT images. The algorithm was trained by micro CT, which is used experimentally due to its very high exposure in spite of high resolution of $\mu \mathrm{m}$ unit. The super-resolution imaging of CBCT showed similar errors of root canal volume and length to those of micro CT. This is expected to provide great assistance to the diagnosis of teeth with complex root canal systems.

In selected studies, detecting various diseases and risk factors in field of dentistry has been attempted using deep learning, such as dental plaque, dental caries, periodontal disease, and periapical disease, as well as the diseases of adjacent anatomical structures observed in dental images such as the maxillary sinusitis, osteoarthritis of the temporomandibular joint, Sjögren syndrome of the parotid gland, and osteoporosis. Kats et al. reported $83 \%$ accuracy when detecting atherosclerotic carotid plaques in panoramic radiographs using an $\mathrm{R}-\mathrm{CNN}{ }^{49}$ This algorithm is expected to help diagnose and treat ischemic brain disease because the plaque is known to be significantly associated with strokes. ${ }^{73}$

When deep learning was applied to the orthodontics, the automatic detection of cephalometric landmarks from radiographs, or facial asymmetry from photographs are expected to shorten the work time of dentists for developing problem list and establishing diagnosis of patients. Meanwhile, deep learning algorithms can improve the fitness, retention, and longevity of the prosthesis by compensating for the deformative errors of 3D printed crowns, detecting abutment margins, and predicting the possibility of the prosthesis falling off.

The study of Luo et al., which classified tooth brushing motions by analyzing 3D motion signals with an RNN-based algorithm collected from wearable devices ${ }^{62}$ shows the potential for personalized dentistry. Personalized feedbacks based on daily obtained data through wearable devices can help patients to reflect and improve oral hygiene themselves.

To summarize the above discussion, utilization of deep learning algorithms is expected to shorten the work time of dentists and ultimately improve treatment results by assisting dentists in almost all aspect of clinical practices such as tooth numbering, disease detection and classification, image quality enhancement, detection of cephalometric landmarks, and the evaluation of prostheses and reduction of errors. Therefore, it is considered that the interest and participation of many dental practitioners is necessary to successfully integrate these technologies into the dentistry. 


\section{Acknowledgement}

This research was supported by a grant of the Korea Health Technology R\&D Project through the Korea Health Industry Development Institute (KHIDI), funded by the Ministry of Health \& Welfare, Republic of Korea (grant number: HI20C0129).

\section{References}

1. Hype cycle. Gartner. https://www.gartner.com/smarterwithgartner/top-trends-on-the-gartner-hypecycle-for-artificial-intelligence-2019/ (2019).

2. Krizhevsky A, Sutskever I, Hinton GE. ImageNet classification with deep convolutional neural networks. In: Advances in neural information processing systems. 2012. p.1097-105.

3. Fukushima K. Neocognitron: a self-organizing neural network model for a mechanism of pattern recognition unaffected by shift in position. Biol Cybern 1980;36:193-202.

4. Hubel DH, Wiesel TN. Receptive fields of single neurones in the cat's striate cortex. J Physiol 1959; 148:574-91.

5. LeCun Y, Bottou L, Bengio Y, Haffner, P. Gradient-based learning applied to document recognition. Proc IEEE 1998;86:2278-323.

6. Ronneberger O, Fischer P, Brox T. U-net: convolutional networks for biomedical image segmentation. In: Lecture Notes in Computer Science (including subseries Lecture Notes in Artificial Intelligence and Lecture Notes in Bioinformatics). Springer; 2015. p.234-41.

7. Ren S, He K, Girshick R, Sun J. Faster R-CNN: towards real-time object detection with region proposal networks. IEEE Trans Pattern Anal Mach Intell 2017;39:1137-49.

8. Suwajanakorn S, Seitz SM, Kemelmacher-Shlizerman I. Synthesizing Obama: learning lip sync from audio. In: ACM Transactions on Graphics 2017;36:1-13.

9. Hochreiter S, Schmidhuber J. Long short-term memory. Neural Comput 1997;9:1735-80.

10. Cho K, Van Merriënboer B, Gulcehre C, Bahdanau D, Bougares F, Schwenk H, et al. Learning phrase representations using RNN encoder-decoder for statistical machine translation. In: EMNLP 2014 - 2014 Conference on Empirical Methods in Natural Language Processing, Proceedings of the Conference. 2014. p.1724-34.

11. Goodfellow I, Pouget-Abadie J, Mirza M, Xu B, Warde-Farley D, Ozair S, et al. Generative adversarial nets. In: Advances in Neural Information Processing Systems. 2014. p.2672-80.

12. Chen H, Zhang K, Lyu P, Li H, Zhang L, Wu J, et al. A deep learning approach to automatic teeth detection and numbering based on object detection in dental periapical films. Sci Rep 2019;9:1-11.

13. Tuzoff DV, Tuzova LN, Bornstein MM, Krasnov AS, Kharchenko MA, Nikolenko SI, et al. Tooth detection and numbering in panoramic radiographs using convolutional neural networks. Dentomaxillofac Radiol 2019;48:20180051.

14. Zhang K, Wu J, Chen H, Lyub P. An effective teeth recognition method using label tree with cascade network structure. Comput Med Imaging Graph 2018;68:61-70.

15. Jader G, Fontineli J, Ruiz M, Abdalla K, Pithon M, Oliveira L. Deep instance segmentation of teeth in panoramic X-ray images. In: 2018 31st SIBGRAPI Conference on Graphics, Patterns and Images (SIBGRAPI). 2018;400-7. 
16. Merdietio Boedi R, Banar N, De Tobel J, Bertels J, Vandermeulen D, Thevissen PW. Effect of lower third molar segmentations on automated tooth development staging using a convolutional neural network. J Forensic Sci 2020;65:481-6.

17. Tian S, Dai N, Zhang B, Yuan F, Yu Q, Cheng X. Automatic classification and segmentation of teeth on 3D dental model using hierarchical deep learning networks. IEEE Access 2019;7:84817-28.

18. Xu X, Liu C, Zheng Y. 3D tooth segmentation and labeling using deep convolutional neural networks. IEEE Trans Vis Comput Graph 2019;25:2336-48.

19. Minnema J, Eijnatten M, Hendriksen AA, Liberton N, Pelt DM, Batenburg KJ, et al. Segmentation of dental cone-beam CT scans affected by metal artifacts using a mixed-scale dense convolutional neural network. Med Phys 2019;46:5027-35.

20. Duong DQ, Nguyen KT, Kaipatur NR, Lou EHM, Noga M, Major PW, et al. Fully automated segmentation of alveolar bone using deep convolutional neural networks from intraoral ultrasound images. In: 2019 41st Annual International Conference of the IEEE Engineering in Medicine and Biology Society (EMBC). 2019. p.6632-5.

21. De Tobel J, Radesh P, Vandermeulen D, Thevissen PW. An automated technique to stage lower third molar development on panoramic radiographs for age estimation: a pilot study. J Forensic Odontostomatol 2017;35:42-54.

22. Du X, Chen Y, Zhao J, Xi Y. A convolutional neural network based auto-positioning method for dental arch in rotational panoramic radiography. In: 2018 40th Annu Int Conf IEEE Eng Med Biol Soc (EMBC). IEEE, 2018. p.2615-8.

23. Liang K, Zhang L, Yang Y, Yang H, Xing Y. A self-supervised deep learning network for low-dose CT reconstruction. In: 2018 IEEE Nuclear Science Symposium and Medical Imaging Conference Proceedings (NSS/MIC). 2018. p.1-4.

24. Hu Z, Jiang C, Sun F, Zhang Q, Ge Y, Yang Y, et al. Artifact correction in low-dose dental CT imaging using Wasserstein generative adversarial networks. Med Phys 2019;46:1686-96.

25. Hegazy MAA, Cho MH, Cho MH, Lee SY. U-net based metal segmentation on projection domain for metal artifact reduction in dental CT. Biomed Eng Lett 2019;9:375-85.

26. Dinkla AM, Florkow MC, Maspero M, Savenije MHF, Zijlstra F, Doornaert PAH, et al. Dosimetric evaluation of synthetic CT for head and neck radiotherapy generated by a patch-based threedimensional convolutional neural network. Med Phys 2019;46:4095-104.

27. Hatvani J, Basarab A, Tourneret J, Gyöngy M, Kouamé D. A tensor factorization method for 3-D super resolution with application to dental CT. IEEE Trans Med Imaging 2019;38:1524-31.

28. Hatvani J, Horváth A, Michetti J, Basarab A, Kouamé D, Gyöngy M. Deep learning-based superresolution applied to dental computed tomography. IEEE Trans Radiat Plasma Med Sci 2019;3: $120-8$.

29. Kumar P, Srivastava MM. Example mining for incremental learning in medical imaging. In: 2018 IEEE Symposium Series on Computational Intelligence (SSCI). 2018. p.48-51.

30. Lee JH, Kim DH, Jeong SN, Choi SH. Detection and diagnosis of dental caries using a deep learningbased convolutional neural network algorithm. J Dent 2018;77:106-11.

31. Casalegno F, Newton T, Daher R, Abdelaziz M, Lodi-Rizzini A, Schürmann F, et al. Caries detection with near-infrared transillumination using deep learning. J Dent Res 2019;98:1227-33.

32. Moutselos K, Berdouses E, Oulis C, Maglogiannis I. Recognizing occlusal caries in dental intraoral images using deep learning. In: 201941 st Annual International Conference of the IEEE Engineering in Medicine and Biology Society (EMBC). 2019. p.1617-20.

33. Prajapati SA, Nagaraj R, Mitra S. Classification of dental diseases using CNN and transfer learning. 
2017 5th Int Symp Comput Bus Intell. IEEE, 2017. p.70-4.

34. Liu L, Xu J, Huan Y, Zou Z, Yeh S-C, Zheng L-R. A smart dental health-IoT platform based on intelligent hardware, deep learning and mobile terminal. IEEE J Biomed Heal Informatics 2019;24:898-906.

35. Bezruk V, Krivenko S, Kryvenko L. Salivary lipid peroxidation and periodontal status detection in ukrainian atopic children with convolutional neural networks. In: 2017 4th International ScientificPractical Conference Problems of Infocommunications. Science and Technology (PIC S\&T). 2017. p.122-4.

36. Aberin STA, De Goma JC. Detecting periodontal disease using convolutional neural networks. In: 2018 IEEE 10th International Conference on Humanoid, Nanotechnology, Information Technology,Communication and Control, Environment and Management (HNICEM). 2018. p.1-6.

37. Joo J, Jeong S, Jin H, Lee U, Yoon JY, Kim SC. Periodontal disease detection using convolutional neural networks. In: 2019 International Conference on Artificial Intelligence in Information and Communication (ICAIIC). 2019. p.360-2.

38. Krois J, Ekert T, Meinhold L, Golla T, Kharbot B, Wittemeier A, et al. Deep learning for the radiographic detection of periodontal bone loss. Sci Rep 2019;9:1-6.

39. Uthoff RD, Song B, Sunny S, Patrick S, Suresh A, Kolur T, et al. Point-of-care, smartphone-based, dual-modality, dual-view, oral cancer screening device with neural network classification for lowresource communities. PLoS One. 2018;13:e0207493.

40. Aubreville M, Knipfer C, Oetter N, Jaremenko C, Rodner E, Denzler J. Automatic classification of cancerous tissue in laserendomicroscopy images of the oral cavity using deep learning. Sci Rep 2017;7:1-10.

41. Forslid G, Wieslander H, Bengtsson E, Wahlby C, Hirsch J-M, Stark CR, et al. Deep convolutional neural networks for detecting cellular changes due to malignancy. In: 2017 IEEE International Conference on Computer Vision Workshops (ICCVW). 2017. p.82-9.

42. Das DK, Bose S, Maiti AK, Mitra B, Mukherjee G, Dutta PK. Automatic identification of clinically relevant regions from oral tissue histological images for oral squamous cell carcinoma diagnosis. Tissue Cell 2018;53:111-9.

43. Jeyaraj PR, Samuel Nadar ER. Computer-assisted medical image classification for early diagnosis of oral cancer employing deep learning algorithm. J Cancer Res Clin Oncol 2019;145:829-37.

44. Ekert T, Krois J, Meinhold L, Elhennawy K, Emara R, Golla T, et al. Deep learning for the radiographic detection of apical lesions. J Endod 2019;45:917-22.e5.

45. Murata M, Ariji Y, Ohashi Y, Kawai T, Fukuda M, Funakoshi T, et al. Deep-learning classification using convolutional neural network for evaluation of maxillary sinusitis on panoramic radiography. Oral Radiol 2019;35:301-7.

46. De Dumast P, Mirabel C, Cevidanes L, Ruellas A, Yatabe M, Ioshida M, et al. A web-based system for neural network based classification in temporomandibular joint osteoarthritis. Comput Med Imaging Graph 2018;67:45-54.

47. Kise Y, Ikeda H, Fujii T, Fukuda M, Ariji Y, Fujita H, et al. Preliminary study on the application of deep learning system to diagnosis of Sjögren's syndrome on CT images. Dentomaxillofac Radiol 2019;48:20190019.

48. Chu P, Bo C, Liang X, Yang J, Megalooikonomou V, Yang F, et al. Using octuplet siamese network for osteoporosis analysis on dental panoramic radiographs. In: 2018 40th Annual International Conference of the IEEE Engineering in Medicine and Biology Society (EMBC). 2018. p.2579-82.

49. Kats L, Vered M, Zlotogorski-Hurvitz A, Harpaz I. Atherosclerotic carotid plaque on panoramic 
radiographs: neural network detection. Int J Comput Dent 2019;22:163-9.

50. Murata S, Lee C, Tanikawa C, Date S. Towards a fully automated diagnostic system for orthodontic treatment in dentistry. In: 2017 IEEE 13th International Conference on e-Science (e-Science). 2017. p.1-8.

51. Patcas R, Bernini DAJ, Volokitin A, Agustsson E, Rothe R, Timofte R. Applying artificial intelligence to assess the impact of orthognathic treatment on facial attractiveness and estimated age. Int J Oral Maxillofac Surg 2019;48:77-83.

52. Leonardi RM, Giordano D, Maiorana F, Greco M. Accuracy of cephalometric landmarks on monitordisplayed radiographs with and without image emboss enhancement. Eur J Orthod 2010;32: 242-7.

53. Qian J, Cheng M, Tao Y, Lin J, Lin H. CephaNet: an improved faster R-CNN for cephalometric landmark detection. 2019 IEEE 16th Int Symp Biomed Imaging (ISBI 2019). 2019. p.868-71.

54. Torosdagli N, Liberton DK, Verma P, Sincan M, Lee JS, Bagci U. Deep geodesic learning for segmentation and anatomical landmarking. IEEE Trans Med Imaging 2019;38:919-31.

55. Shen Z, Shang X, Li Y, Bao Y, Zhang Xj, Dong X, et al. PredNet and CompNet: prediction and highprecision compensation of in-plane shape deformation for additive manufacturing. In: 2019 IEEE 15th International Conference on Automation Science and Engineering (CASE). 2019. p.462-7.

56. Yamaguchi S, Lee C, Karaer O, Ban S, Mine A, Imazato S. Predicting the debonding of CAD/CAM composite resin crowns with AI. J Dent Res 2019;98:1234-8.

57. Zhang B, Dai N, Tian S, Yuan F, Yu Q. The extraction method of tooth preparation margin line based on S-Octree CNN. Int J Numer Method Biomed Eng 2019;35:e3241.

58. Zhao M, Xiong G, Shang X, Liu C, Shen Z, Wu H. Nonlinear deformation prediction and compensation for 3D printing based on CAE neural networks. In: 2019 IEEE 15th International Conference on Automation Science and Engineering (CASE). 2019. p.667-72.

59. Milošević D, Vodanović M, Galić I, Subašić M. Estimating biological gender from panoramic dental X-ray images. In: 2019 11th International Symposium on Image and Signal Processing and Analysis (ISPA). 2019. p.105-10.

60. Ilić I, Vodanović M, Subašić M. Gender estimation from panoramic dental X-ray images using deep convolutional networks. In: IEEE EUROCON 2019 -18th International Conference on Smart Technologies. 2019. p.1-5.

61. Ali H, Khursheed M, Fatima SK, Shuja SM, Noor S. Object recognition for dental instruments using SSD-MobileNet. In: 2019 International Conference on Information Science and Communication Technology (ICISCT). 2019. p.1-6.

62. Luo C, Feng X, Chen J, Li J, Xu W, Li W, et al. Brush like a dentist: accurate monitoring of toothbrushing via wrist-worn gesture sensing. In: IEEE INFOCOM 2019 - IEEE Conference on Computer Communications. 2019. p.1234-42.

63. Chollet F. Deep learning mit python und keras: das praxis-handbuch vom entwickler der kerasbibliothek. MITP-Verlags GmbH \& Co. KG. 2018.

64. Mcculloch WS, Pitts W. A logical calculus nervous activity. Bull Math Biol 1990;52:99-115.

65. Rosenblatt F. The perceptron: a probabilistic model for information storage and organization in the brain. Psychol Rev 1958;65:386-408.

66. Minsky M, Papert S. Perceptron: an introduction to computational geometry. MIT press; 2017.

67. Werbos PJ. The roots of backpropagation: from ordered derivatives to neural networks and political forecasting. John Wiley \& Sons; 1994.

68. Rumelhart DE, Hinton GE, Williams RJ. Learning representations by back-propagating errors. 
Nature. 1986;323:533-6.

69. Glorot X, Bordes A, Bengio Y. Deep sparse rectifier neural networks. In: Proceedings of the fourteenth international conference on artificial intelligence and statistics. 2011. p.315-23.

70. Hinton GE, Osindero S, Teh YW. A fast learning algorithm for deep belief nets. Neural Comput 2006;18:1527-54.

71. Glorot X, Bengio Y. Understanding the difficulty of training deep feedforward neural networks. In: Proceedings of the thirteenth international conference on artificial intelligence and statistics. 2010. p.249-56.

72. He K, Zhang X, Ren S, Sun J. Delving deep into rectifiers: surpassing human-level performance on imagenet classification. In: Proceedings of the IEEE international conference on computer vision. 2015. p.1026-34.

73. Takaya N, Yuan C, Chu B, Saam T, Underhill H, Cai J, et al. Association between carotid plaque characteristics and subsequent ischemic cerebrovascular events: a prospective assessment with MRI - initial results. Stroke 2006;37:818-23.

74. Eun H, Kim C. Oriented tooth localization for periapical dental X-ray images via convolutional neural network. In: 2016 Asia-Pacific Signal and Information Processing Association Annual Summit and Conference (APSIPA). 2016. p.1-7.

75. Oktay AB. Tooth detection with convolutional neural networks. 2017 Med Technol Natl Conf TIPTEKNO 2017. 2017. p.1-4.

76. Miki Y, Muramatsu C, Hayashi T, Zhou X, Hara T, Katsumata A, et al. Classification of teeth in cone-beam CT using deep convolutional neural network. Comput Biol Med 2017;80:24-9.

77. Koch TL, Perslev M, Igel C, Brandt SS. Accurate segmentation of dental panoramic radiographs with U-nets. In: 2019 IEEE 16th International Symposium on Biomedical Imaging (ISBI 2019). 2019. p.15-9.

78. Hiraiwa T, Ariji Y, Fukuda M, Kise K, Nakata K, Katsumata A, et al. A deep-learning artificial intelligence system for assessment of root morphology of the mandibular first molar on panoramic radiography. Dentomaxillofac Radiol 2019;48:20180218.

79. Vinayahalingam S, Xi T, Berge S, Maal T, de Jong G. Automated detection of third molars and mandibular nerve by deep learning. Sci Rep 2019;9:1-7.

80. Yauney G, Angelino K, Edlund D, Shah P. Convolutional neural network for combined classification of fluorescent biomarkers and expert annotations using white light images. In: 2017 IEEE 17th International Conference on Bioinformatics and Bioengineering (BIBE). 2017. p.303-9.

81. Rana A, Yauney G, Wong LC, Gupta O, Muftu A, Shah P. Automated segmentation of gingival diseases from oral images. In: 2017 IEEE Healthcare Innovations and Point of Care Technologies (HI-POCT). 2017. p.144-7.

82. Yang J, Xie Y, Liu L, Xia B, Cao Z, Guo C. Automated dental image analysis by deep learning on small dataset. In: 2018 IEEE 42nd Annual Computer Software and Applications Conference (COMPSAC). 2018. p.492-7.

83. Song T, Landini G, Fouad S, Mehanna H. Epithelial segmentation from in situ hybridisation histological samples using a deep central attention learning approach. In: 2019 IEEE 16th International Symposium on Biomedical Imaging (ISBI 2019). 2019. p.1527-31.

84. Shen Z, Shang X, Zhao M, Dong X, Xiong G, Wang F-Y. A learning-based framework for error compensation in 3d printing. IEEE Trans Cybern 2019;49:4042-50.

85. Alarifi A, AlZubi AA. Memetic search optimization along with genetic scale recurrent neural network for predictive rate of implant treatment. J Med Syst 2018;42:202. 


\section{Supplement 1. Excluded study and reason for exclusion}

\section{Author Year Reason for exclusion}

Colchester 1992

No information on algorithm performance evaluation

Economopoulos 2008

It is hard to consider that the enhanced hexagonal centre-based inner search algorithm proposed in this paper is included in deep learning.

Han 2017

No information on algorithm performance evaluation

Hu 2019

A study to evaluate the brain's perception of cold stimulation

Mendoca 2004

No information on algorithm performance evaluation

Yoon 2018

Research using deep learning for data mining 